\title{
A resource-task network approach for optimal short-term/periodic scheduling and heat integration in multipurpose batch plants
}

\author{
Cheng-Liang Chen ${ }^{*}$, Chia-Yuan Chang \\ Department of Chemical Engineering, National Taiwan University, No. 1, Section 4, Roosevolt Road, Taipei 10617, Taiwan, ROC
}

\section{A R T I C L E I N F O}

\section{Article history:}

Received 18 September 2007

Accepted 11 June 2008

Available online $\mathrm{xxxx}$

\section{Keywords:}

Multi-purpose batch processes

Short-term scheduling

Periodic scheduling

Heat integration

Mixed-integer linear program (MILP)

\begin{abstract}
A B S T R A C T
This article aims to integrate the task scheduling and heat recovery problems into an unified framework for multi-purpose batch processes. The batch scheduling formulation is extended from the continuous Resource-Task Network (RTN) formulation which was originally proposed by Castro et al. [P.M. Castro, A.P. Barbosa-Póvoa, H.A. Matos, Optimal periodic scheduling of batch plants using RTN-based discrete and continuous-time formulations: a case study approach, Ind. Eng. Chem. Res. 42 (2003) 3346-3360; P.M. Castro, A.P. Barbosa-Póvoa, H.A. Matos, A.Q. Novais, Simple continuous-time formulation for short-term scheduling of batch and continuous processes, Ind. Eng. Chem. Res. 43 (2004) 105-118]. Besides the integrated heat recovery and short-term batch scheduling, a full set of heat-integrated periodic scheduling for batch processes, which is more useful in industrial environments, is also presented. The heat-integrated batch scheduling problem can be formulated as a mixed-integer linear program (MILP), where the new formulation can also be solved in standalone or heat-integrated modes by user's preferences. In the heat-integrated mode, the processes can be precisely defined while keeping the operation flexibility with slightly expanded model size. Two literature examples are demonstrated to show the efficiency and flexibility of the model.
\end{abstract}

(ㄱ) 2008 Elsevier Ltd. All rights reserved.

\section{Introduction}

Batch production processes have brought more and more attention during the past decades because of the growing requirements for small amount but high-value added products. Though batch operations seldom require significant amount of energy compared to their continuous counterparts, as mentioned by Lee and Reklaitis [3] and Vaklieva-Bancheva and Ivanov [4], suitable scheduling of operation order may reduce the demand of energy in the long run. Furthermore, partly because of the lack of heat recovery in batch processes, it is sometimes far more likely to perform heat integration to save energy in batch plants [5]. Simultaneous consideration of production scheduling and heat recovery opportunity thus become an attractive subject in batch plants.

Until recently, not many studies made the connection between production scheduling and heat integration in batch processes. In the earlier stage, there were implicitly predefined schedules and the major efforts were focused on the analysis of heat exchanger network (HEN) which target at the minimum utility, the number of heat exchange units and temperature of the inlet and outlet streams, etc. Considerable effort and progress has been made in the development of insight-based pinch analysis techniques and

\footnotetext{
* Corresponding author. Tel.: +886 2 23636194; fax: +886 223623040 .

E-mail address: CCL@ntu.edu.tw (C.-L. Chen).
}

mathematical optimization of super-structure, respectively. Most of these works were developed for both continuous and batch processes (which were usually adapted from their continuous counterparts), in which, however, production scheduling was of little concern.

A pioneered study of the heat recovery for batch processes is done by Vaselanak et al. [6]. They designed a heuristic pairing rule for one-by-one matching of the heat and cold tank but the exchanging time is assumed to be carried out at the implicit predefined schedule. Vaklieva-Bancheva and Ivanov [4] purposed a MILP formulation for heat exchange network design for multipurpose batch plants where direct heat exchange is considered. In their later work [7], a single level formulation that selects the production campaigns and the design of cost-optimal heat exchanger network simultaneously is addressed. The main limitation of the formulation is that each batch stage must operates in a zero-wait mode with a fixed processing time. On the basis of this work, Bozan et al. [8] further developed a single step, interactive user-friendly computer program (BatcHEN) to determine the campaigns, heat exchanger areas of potential heat exchangers for multipurpose batch chemical plants.

In most earlier techniques that were developed for batch heat integration, it is usually assumed that production schedule is given a priori. This assumption is somehow understandable in the early stage of technique development, as all methods have to start from 


\section{Notations}

$\begin{array}{ll}\text { Sets } & \\ \mathscr{I} & \text { process tasks } \\ \mathscr{I}^{b} & \text { batch tasks } \\ \mathscr{I}^{Z W} & \text { batch tasks with zero-wait } \\ \mathscr{I}_{C}^{b} & \text { batch tasks that requires cooling } \\ \mathscr{I}_{H}^{b} & \text { batch tasks that requires heating } \\ \mathscr{R}^{b} & \text { process resources } \\ \mathscr{R}^{\mathrm{RM}} & \text { raw material resources } \\ \mathscr{R}^{\mathrm{FP}} & \text { final product resources } \\ \mathscr{R}^{\mathrm{EQ}} & \text { equipment resources } \\ \mathscr{T} & \text { time points }\end{array}$

\section{Parameters}

$c_{r} \quad$ selling price of resource $r$

$c_{i} / \bar{c}_{i} \quad$ constant/proportional cost of running task $i$

$c_{H} / c_{C} \quad$ unit cost of steam/cooling water

$H^{\text {min }} / H^{\max }$ minimum/maximum of time horizon for shot-term scheduling or cycle time for periodic scheduling

$K_{1, i}, K_{2, i}$ coefficient for external utility requirements in task $i$ (standalone mode)

$K_{1, i}^{\prime}, K_{2, i}^{\prime}$ coefficient for external utility requirements in task $i$ (heat-int. mode)

$R_{r}^{\min } / R_{r}^{\max }$ minimum/maximum availability of resource $r$

$R_{r}^{0} \quad$ initial available of resource $r$

$V_{i}^{\min } / V_{i}^{\max }$ minimum/maximum amount of material processed by task $i$

$\alpha_{i} / \beta_{i} \quad$ constant/proportional term in the processing time of task $i$

$\mu_{r, i}, \bar{\mu}_{r, i} \quad$ coefficient for the equipment resource $r$ at the start/end of task $i$ $v_{r}, i / \bar{v}_{r, i} \quad$ coeff. for the material resource $r$ at the start/end of task $i$

$\Delta \alpha_{i} \quad$ incremental processing time in heat-integrated mode

$\Delta \alpha_{i}^{\prime} \quad$ incremental processing time in computing heat duty in heat-integrated mode

$\Delta t \quad$ maximum number of event points allowed between the beginning and end of a given task

$\delta_{i} / \delta_{i}^{\prime} \quad$ number of time point right shifted from the beginning/ end of its corresponding heat-integrated task $i$

$\Delta_{r}^{\min } / \Delta_{r}^{\max }$ minimum/maximum on the demand rate of resource $r$

Binary variables

$\bar{N}_{i, t, t^{\prime}} \quad=1$ if a task $i$ takes place at time point $t$ to $t^{\prime}$

$x_{i, i^{\prime}, t, t^{\prime}}=1$ if there is a heat integration between task $i$ and $i^{\prime}$ at time point $t$ to $t^{\prime}$

Continuous variables

$\mathrm{H}$ time horizon for shot-term scheduling or cycle time for periodic scheduling

$q_{i, t, t^{\prime}} \quad$ amount of external utility required by task $i$ (standalone mode)

$q_{i, t, t^{\prime}}^{\prime} \quad$ amount of external utility required by task $i$ (heat-integrated mode)

$Q_{H} / Q_{C} \quad$ total required steam/cooling water

$R_{r, t} \quad$ excess amount of resource $r$ at event point $t$

$T_{t} \quad$ absolute time of event point $t$

$\bar{\xi}_{i, t, t^{\prime}} \quad$ total amount of material processed by task $i$ at time point $t$ to $t^{\prime}$

$\Delta r \quad$ demand rate of resource $r$ for each cycle a manageable level. This assumption was relaxed in the later stage where several works reported the inclusion of process rescheduling by pinch-based researchers $[9,10]$. To combine heat integration with scheduling, Papageorgiou et al. [11] presented a general framework, which extends the State-Task Network (STN) originally proposed by Kondili et al. [12] to take both direct and indirect heat integrations into account with scheduling. The formulation is based on discretizing time horizon into a number of intervals of equal duration. Lee and Reklaitis [3] presented an optimal periodic scheduling with direct heat integration. In their work, it is assumed that the production occurs as a series of batches and results in a multistage and single product plant. Barbosa-Póvoa et al. [13] presented a mathematical formulation for detailed design of multi-purpose batch facilities where heat-integration feature were addressed at the design level. The problem is formulated in a discrete-time framework based on the STN and the maximal State-Task Network (mSTN) to represent the interactions between the process and equipment. Pinto et al. [14] extended the previous work and proposed a mathematical formulation for the detailed design of multi-purpose batch process facilities where direct heat integration and economic savings in utilities are considered simultaneously. In their work, both capital investment within superstructure level and the potential of heat integration in the operation level results in significant savings. Majozi [15] proposed a continuous formulation for optimization of heat-integrated batch chemical plants. Therein the direct heat-integration assumption is also applied. Due to the framework of extension of the State-Sequence-Network (SSN) proposed by Majozi and Zhu [16], the system is capable of dealing with both multi-product and multipurpose batch processes with very few binary variables. One shortcoming of this work is that only short-term problems are investigated.
The main goal of this work is to present a more complete framework for directly heat-integrated multi-purpose batch plants. The formulation in direct heat integration is transplanted from the work by Majozi [15] with extensions for more general cases. The scheduling engine we applied is the continuous Resource-Task Network (RTN) framework proposed by Castro et al. [1,2], the reason for applying which is its compact and simple form. Shaik et al. [17] have also noted that the time-grid-based model by Castro et al. is one of the best single time grid formulations for short-term scheduling of multi-purpose batch plants.

The rest of this paper is organized as follows: Section 2 involves the problem description for batch scheduling with direct heat integration in short-term and periodic operation modes; Section 3 provides the mathematical formulation; in Section 4, two literature examples are presented, showing the proposed framework's flexibility in various heat-exchanging conditions. Conclusions are finally made in Section 5.

\section{Problem statement}

The scheduling problems with heat integration discussed in this article can be classified into two main types: the short-term and the periodic modes. In both scheduling modes, the following information must be given:

(i) Processing recipe that involves a set of operations, i.e., processing times, coefficients for resources consumption/ generation.

(ii) A set of available equipments and initial available resources.

(iii) Hot and cold duties for units that require heating and cooling. 
(iv) Cost of materials, utilities and operation for each task, selling price of products.

In this work, a given time horizon is applied in short-term problem because the goal is to maximize the overall profit. In periodic operation mode, it is more attractive to find the optimal profit per cycle time because we usually prefer a long-term operation in this mode. It is noted that the optimal schedule obtained in the periodic mode contains only repeating tasks. Since the target for periodic scheduling is on a long-term basis, the time required for start-up and shutdown are short and can be omitted. However, it is practicable to introduce an optimal short-term scheduling in the start-up and shutdown procedure to approximate a more economical overall result. After optimization, the desirable number of runs in every unit can be determined.

\section{Mathematical formulation}

Time representation, discrete or continuous, is the most important issue in batch scheduling. In discrete formulation, time horizon is divided into numerous time intervals by predefining locations of time points that are usually equally spaced. The imprecise nature leads to not only an inaccurate solution but also a larger problem size. Hence many subsequent attempts are dedicated to efficiently allocating time points from the information of task durations. These approaches are categorized as the continuous type. A recently comprehensive comparison between discrete and continuous time models was drawn by Floudas and Lin [18]. Different continuous time representations for batch scheduling problems have also been reviewed by Shaik et al. [17] and Méndez et al. [19]. It is worth-noting that no matter what kind of continuous model is chosen, the reason why continuous model is superior to conventional discrete model is the fewer number of time points. In this connection, the continuous characteristics would raise the complexity of model but the number of binary variables can be reduced remarkably. In this article, the continuous RTN formulation proposed by Castro et al. [1,2] is extended to consider direct heat integration. As mentioned by Shaik et al. [17], the continuous RTN is categorized as a global-event-based continuous-time formulation which uses a set of events (time points) that are common across all tasks. In other words, it is allowable for a task crossing some time points because the time points are commonly used.

In order to make it easier to understand the mathematical formulation, the time representation of short-term and periodic scheduling is shown in Fig. 1. It should be reminded that the time horizon represented in periodic version is cycle time. The original continuous RTN representation can be found in the works done by Castro et al. [1,2]. To have a consistent mapping with short-term formulation, the last time point chosen is $T$ rather than $T+1$ as in the work done by Castro et al. In other words, either short-term or periodic operation, the full time horizon (or cycle time) is divided into $T$ time points. It is also observed that the last time point refers to the first time point for periodic operation. The following two subsections will illustrate formulations for short-term and periodic operations, respectively.

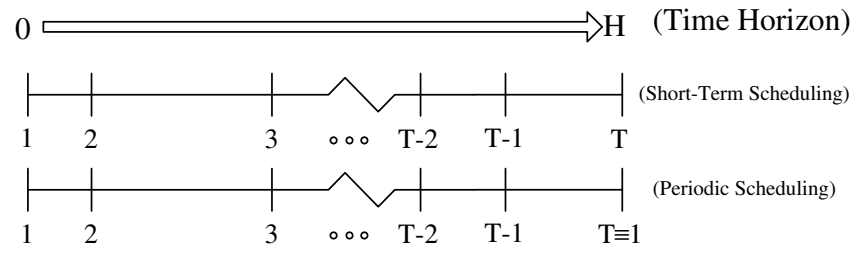

Fig. 1. Continuous time representation for two scheduling modes in this work.

\subsection{Formulation for short-term scheduling problems}

The MILP formulation for short-term scheduling problems will be established first, revised formulations for periodic scheduling then follows in the next subsection. Several constraints should be considered for heat-integrated sort-term scheduling problems, including tasks timings, operations, capacities, and resource balances.

\subsubsection{Time constraints}

Eq. (1) shows the timing relationship between an absolute time of time point $t\left(T_{t}\right)$ with its adjacent time points spanned from $t$ to $t+\Delta t$ for an equipment resource $r \in R^{\mathrm{EQ}}$. The parameter $\Delta t$ specifies the maximum number of time points allowed between the beginning and the end of a task. $\bar{\mu}_{r, i}$ is the coefficient of generation which maps equipment resource $r$ to task $i$ at the end of that task. For simplicity purpose, if a task can be done in more than one equipment, an equivalent number of task must be defined (i.e., decoupling of resource and task). Hence, the coefficients outside the square bracket ( 1 or 0 ) denote the relative tasks that must be considered whereas the three terms in the square bracket represent their processing times. The first term in the summation refers to the constant processing time (denoted as $\alpha_{i}$ ); the third term refers to the processing time which is directly proportional to the corresponding working amount (denoted as $\beta_{i} \bar{\xi}_{i, t, t^{\prime}}$ ). The second term refers to an increment of processing time (denoted as $\Delta \alpha_{i}$ ). Here we consider the processing may last longer in heat-integration mode due to some reasons, such as lower converting efficiency in heat integration or used by performing cleaning operations. The binary variable $x_{i, i^{\prime}, t, t^{\prime}}$ denotes whether a task $i$, which requires heating, is integrated with a task $i^{\prime}$, which requires cooling, at the time interval $t$ to $t^{\prime}$ It is worth-noting that since we do not known a priori whether an utility required by a task in heat integration is hot or cold, two local summations must be added together to confirm its effect.

More comprehension of one-to-one heat integration is illustrated in Section 3.1.5.

$$
\begin{aligned}
T_{t^{\prime}}-T_{t} \geqslant & \sum_{i \in \mathscr{I}^{b}} \bar{\mu}_{r, i}\left[\alpha_{i} \bar{N}_{i, t, t^{\prime}}+\Delta \alpha_{i}\left(\sum_{i^{\prime} \in \mathscr{J}_{C}^{b}} x_{i, i^{\prime}, t+\delta_{i}, t^{\prime}+\delta_{i}^{\prime}}+\sum_{i^{\prime} \in \mathscr{S}_{H}^{b}} x_{i^{\prime}, i, t+\delta_{i}, t^{\prime}+\delta_{i}^{\prime}}\right)\right. \\
& \left.+\beta_{i} \bar{\xi}_{i, t, t^{\prime}}\right] \quad \forall r \in \mathscr{R}^{\mathrm{EQ}}, t \in \mathscr{T}, t^{\prime} \in \mathscr{T}, t< \\
& t^{\prime} \leqslant t+\Delta t, t \neq T
\end{aligned}
$$

Eq. (2) is complementary to Eq. (1) when the corresponding tasks work in zero-wait mode only. Here, zero-wait implies that when a task terminates, all material resources (whether they are intermediates or products) must leave the equipment resource (probably a reactor) immediately. One may notice that if one task is designated as zero-wait, a time point will be allocated at the end of the task whether or not there is another task starting at that time.

$$
\begin{aligned}
T_{t^{\prime}}-T_{t} \leqslant & H^{\max }\left(1-\sum_{i \in \mathscr{I}^{b}, \mathscr{I}^{Z W W}} \bar{\mu}_{r, i} \bar{N}_{i, t, t^{\prime}}\right) \\
& +\sum_{i \in \mathscr{I}^{b}, \mathscr{F}^{Z} W} \bar{\mu}_{r, i}\left[\alpha_{i} \bar{N}_{i, t, t^{\prime}}+\Delta \alpha_{i}\left(\sum_{i^{\prime} \in \mathscr{I}_{C}^{b}} x_{i, i^{\prime}, t+\delta_{i}, t^{\prime}+\delta_{i}^{\prime}}\right.\right. \\
& \left.\left.+\sum_{i^{\prime} \in \mathscr{I}_{H}^{b}} x_{i^{\prime}, i, t+\delta_{i}, t^{\prime}+\delta_{i}^{\prime}}\right)+\beta_{i} \bar{\xi}_{i, t, t^{\prime}}\right] \quad \forall r \in \mathscr{R}^{\mathrm{EQ}}, t, t^{\prime} \in \mathscr{T}, \\
& <t^{\prime} \leqslant t+\Delta t, t \neq T
\end{aligned}
$$

Of course, the first and final time points must be mapped to the real time 0 and time horizon, which are represented in Eqs. (3) and (4), respectively. 
$T_{1}=0$

$T_{T}=H$

The time horizon must be confined to the region of interesting. However, if the objective function is to maximize the profit, it is usually to fix the time horizon at a certain value (i.e., a parameter). That means Eq. (5) is important only if the objective function is minimizing time horizon or earliness.

$H^{\min } \leqslant H \leqslant H^{\max }$

\subsubsection{Operational constraints}

In batch operation mode, the material processed by any task $i$ from $t$ to $t^{\prime}$ is simply expressed by Eq. (6). If a task $i$ is not taken place at that time period, it is reasonable to fix the $\bar{\xi}_{i, t, t^{\prime}}$ as zero.

$$
\begin{aligned}
V_{i}^{\min } \bar{N}_{i, t, t^{\prime}} \leqslant \bar{\xi}_{i, t, t^{\prime}} \leqslant V_{i}^{\max } \bar{N}_{i, t, t^{\prime}} \\
\forall i \in \mathscr{I}^{b}, t, t^{\prime} \in \mathscr{T} \\
t<t^{\prime} \leqslant t+\Delta t, t \neq T
\end{aligned}
$$

\subsubsection{Excess resource balance constraints}

Resource is a general concept that includes not only equipment but also the state of materials in the RTN. Eq. (7) presents the mass balance for the amount of excess resource $R_{r, t}$. The equation includes three parts. First, the excess properties accumulated from previous time point $t-1$ or the initial value of the resource if $t=1$; second, the disappearing amount of resource from $t$ to $t+\Delta t$; third, the generation of resource from $t-\Delta t$ to $t$.

$$
\begin{aligned}
R_{r, t}= & \left.R_{r}^{0}\right|_{t=1}+\left.R_{r, t-1}\right|_{t>1}+\sum_{i \in \mathscr{P}^{b}}\left[\sum_{\substack{t^{\prime} \in \mathscr{T} \\
t<t^{\prime} \leqslant t+\Delta t}}\left(\mu_{r, i} \bar{N}_{i, t, t^{\prime}}+v_{r, i} \bar{\xi}_{i, t, t^{\prime}}\right)\right. \\
& \left.+\sum_{\substack{t^{\prime} \in \mathscr{T} \\
t-\Delta t \leqslant t^{\prime}<t}}\left(\bar{\mu}_{r, i} \bar{N}_{i, t^{\prime}, t}+\bar{v}_{r, i} \bar{\xi}_{i, t^{\prime}, t}\right)\right] \quad \forall r \in \mathscr{R}, t \in \mathscr{T}
\end{aligned}
$$

\subsubsection{Capacity constraints}

For raw material resources, the excess quantity must be hold in the unit or storage tank. Eq. (8) gives an overall constraint in representing resource arrangement. It is noticed that if no intermediate storage (NIS) policy is applied for certain material, these excess resources are simply set to be zero (i.e., $R_{r}^{\max }=0$ ). For equipment resources, due to the decoupling of resource and task, the maximum availability is always 1 .

$R_{r}^{\min } \leqslant R_{r, t} \leqslant R_{r}^{\max } \quad \forall r \in \mathscr{R}, t \in \mathscr{T}$

\subsubsection{Heat-integration constraints}

Direct heat integration between two tasks is possible if and only if they (both heat and cold) cover the same time interval. The binary variable $x_{i, i^{\prime}}$ is applied as an appointment of heat integration for task $i$ with another task $i^{\prime}$ from a shifted number of time points right after the time point $t\left(\delta_{i}\right)$ and terminate at the time $t^{\prime}$ but biased with $\delta_{i}^{\prime}$. In ordinary cases, the two parameters are zero, representing that heat integration is completely synchronous with the task. However, the two shifting parameters open up the opportunities when heat integration must be applied in special conditions.

3.1.5.1. One-to-one constraints. From Eqs. (9) and (10), there are three implications worth to keep in mind: first, it shows that heat integration can only take place if and only if a real task $\bar{N}_{i, t, t^{\prime}}$ occurs from time point $t$ to $t^{\prime}$. Second, the heat-exchanging is always one by one, one task that requires heating $\left(\mathscr{I}_{H}^{b}\right)$ versus to one task that re- quires cooling $\left(\mathscr{I}_{C}^{b}\right)$. It is assumed that hot tasks can exchange heat with cold tasks only. This implies the heat exchange between two hot or two cold tasks are not allowable. In addition, to avoid duplicating use of binary variables in $x_{i, i^{\prime}}$ the task $i \in \mathscr{I}_{H}^{b}$ is always placed as the first index and the task $i \in \mathscr{I}_{C}^{b}$ is always the second index.

$$
\begin{aligned}
& \sum_{i^{\prime} \in \mathscr{I}_{C}^{b}} x_{i, i^{\prime}, t+\delta_{i}, t^{\prime}+\delta_{i}^{\prime}} \leqslant \bar{N}_{i, t, t^{\prime}} \forall i \in \mathscr{I}_{H}^{b}, t, t^{\prime} \in \mathscr{T}, t<t^{\prime} \leqslant t+\Delta t, t \neq T \\
& \sum_{i \in \mathscr{I}_{H}^{b}} x_{i, i^{\prime}, t+\delta_{i^{\prime}}, t^{\prime}+\delta_{i^{\prime}}^{\prime}} \leqslant \bar{N}_{i^{\prime}, t, t^{\prime}} \forall i^{\prime} \in \mathscr{I}_{C}^{b}, t, t^{\prime} \in \mathscr{T}, t<t^{\prime} \leqslant t+\Delta t, t \neq T
\end{aligned}
$$

3.1.5.2. External utility requirement. The paragraph involves the calculation of external utilities required for the tasks that need heating or cooling. As shown in Eqs. (11)-(14), the total amount of consumed steam or cooling water is proportional to the processing time and the amount of material processed. For simplification, it is assumed that only the amount of material processed makes a direct and major contribution to the amount of utilities required whereas it is not significant to the process duration. In this connection, the processing time can be simply expressed as $\alpha_{i}$ (or $\alpha_{i}+\Delta \alpha_{i}^{\prime}$ for adjustment purpose in integrated mode), which maintains the formulation in MILP.

Heat duty required in task $i$ at time points $t$ to $t^{\prime}$ (standalone mode)

$$
\begin{aligned}
q_{i, t, t^{\prime}}= & K_{1, i} \alpha_{i}\left(\bar{N}_{i, t, t^{\prime}}-\sum_{i^{\prime} \in \mathscr{S}_{C}^{b}} x_{i, i^{\prime}, t+\delta_{i}, t^{\prime}+\delta_{i}^{\prime}}\right) \\
& +K_{2, i} \alpha_{i} \bar{\xi}_{i, t, t^{\prime}}\left(\bar{N}_{i, t, t^{\prime}}-\sum_{i^{\prime} \in \mathscr{I}_{C}^{b}} x_{i, i^{\prime}, t+\delta_{i}, t^{\prime}+\delta_{i}^{\prime}}\right) \\
= & K_{1, i} \alpha_{i}\left(\bar{N}_{i, t, t^{\prime}}-\sum_{i^{\prime} \in \mathscr{I}_{C}^{b}} x_{i, i^{\prime}, t+\delta_{i}, t^{\prime}+\delta_{i}^{\prime}}\right)+K_{2, i} \alpha_{i}\left[\Gamma_{1}\left(i, t, t^{\prime}\right)-\Gamma_{2}\left(i, t, t^{\prime}\right)\right] \\
& \forall i \in \mathscr{I}_{H}^{b}, t, t^{\prime} \in \mathscr{T}, t<t^{\prime} \leqslant t+\Delta t, t \neq T
\end{aligned}
$$

Heat duty required in task $i$ at time points $t$ to $t^{\prime}$ (integrated mode)

$$
\begin{aligned}
q_{i, t, t^{\prime}}^{\prime}= & K_{1, i}^{\prime}\left(\alpha_{i}+\Delta \alpha_{i}^{\prime}\right) \sum_{i^{\prime} \in \mathscr{I}_{C}^{b}} x_{i, i^{\prime}, t+\delta_{i}, t^{\prime}+\delta_{i}^{\prime}} \\
& +K_{2, i}^{\prime}\left(\alpha_{i}+\Delta \alpha_{i}^{\prime}\right) \bar{\xi}_{i, t, t^{\prime}} \sum_{i^{\prime} \in \mathscr{I}_{C}^{b}} x_{i, i^{\prime}, t+\delta_{i}, t^{\prime}+\delta_{i}^{\prime}} \\
= & K_{1, i}^{\prime}\left(\alpha_{i}+\Delta \alpha_{i}^{\prime}\right) \sum_{i^{\prime} \in \mathscr{I}_{C}^{b}} x_{i, i^{\prime}, t+\delta_{i}, t^{\prime}+\delta_{i}^{\prime}}+K_{2, i}^{\prime}\left(\alpha_{i}+\Delta \alpha_{i}^{\prime}\right) \Gamma_{2}\left(i, t, t^{\prime}\right) \\
& \forall i \in \mathscr{I}_{H}^{b}, t, t^{\prime} \in \mathscr{T}, t<t^{\prime} \leqslant t+\Delta t, t \neq T
\end{aligned}
$$

Cold duty required in task $i^{\prime}$ at time points $t$ to $t^{\prime}$ (standalone mode)

$$
\begin{aligned}
q_{i^{\prime}, t, t^{\prime}}= & K_{1, i^{\prime}} \alpha_{i^{\prime}}\left(\bar{N}_{i^{\prime}, t, t^{\prime}}-\sum_{i \in \mathscr{S}_{H}^{b}} x_{i, i^{\prime}, t+\delta_{i^{\prime}}, t^{\prime}+\delta_{i^{\prime}}^{\prime}}\right) \\
& +K_{2, i^{\prime}} \alpha_{i^{\prime}} \bar{\xi}_{i^{\prime}, t, t^{\prime}}\left(\bar{N}_{i^{\prime}, t, t^{\prime}}-\sum_{i \in \mathscr{I}_{H}^{b}} x_{i, i^{\prime}, t+\delta_{i^{\prime}}, t^{\prime}+\delta_{i^{\prime}}^{\prime}}\right) \\
= & K_{1, i^{\prime}} \alpha_{i^{\prime}}\left(\bar{N}_{i^{\prime}, t, t^{\prime}}-\sum_{i \in \mathscr{S}_{H}^{b}} x_{i, i^{\prime}, t+\delta_{i^{\prime}}, t^{\prime}+\delta_{i^{\prime}}^{\prime}}\right) \\
& +K_{2, i^{\prime}} \alpha_{i^{\prime}}\left[\Gamma_{3}\left(i^{\prime}, t, t^{\prime}\right)-\Gamma_{4}\left(i^{\prime}, t, t^{\prime}\right)\right] \\
& \forall i^{\prime} \in \mathscr{I}_{C}^{b}, t, t^{\prime} \in \mathscr{T}, t<t^{\prime} \leqslant t+\Delta t, t \neq T
\end{aligned}
$$

Cold duty required in task $i^{\prime}$ at time points $t$ to $t^{\prime}$ (integrated mode) 


$$
\begin{aligned}
q_{i^{\prime}, t, t^{\prime}}^{\prime}= & K_{1, i^{\prime}}^{\prime}\left(\alpha_{i^{\prime}}+\Delta \alpha_{i^{\prime}}^{\prime}\right) \sum_{i \in \mathscr{S}_{H}^{b}} x_{i, i^{\prime}, t+\delta_{i^{\prime}}, t^{\prime}+\delta_{i^{\prime}}^{\prime}} \\
& +K_{2, i^{\prime}}^{\prime}\left(\alpha_{i^{\prime}}+\Delta \alpha_{i^{\prime}}^{\prime}\right) \bar{\xi}_{i^{\prime}, t, t^{\prime}} \sum_{i \in \mathscr{I}_{H}^{b}} x_{i, i^{\prime}, t+\delta_{i^{\prime}}, t^{\prime}+\delta_{i^{\prime}}^{\prime}} \\
= & K_{1, i^{\prime}}^{\prime}\left(\alpha_{i^{\prime}}+\Delta \alpha_{i^{\prime}}^{\prime}\right) \sum_{i \in \mathscr{S}_{H}^{b}} x_{i, i^{\prime}, t+\delta_{i^{\prime}}, t^{\prime}+\delta_{i^{\prime}}^{\prime}}+K_{2, i^{\prime}}^{\prime}\left(\alpha_{i^{\prime}}+\Delta \alpha_{i^{\prime}}^{\prime}\right) \Gamma_{4}\left(i, t, t^{\prime}\right) \\
& \forall i^{\prime} \in \mathscr{I}_{C}^{b}, t, t^{\prime} \in \mathscr{T}, t<t^{\prime} \leqslant t+\Delta t, t \neq T
\end{aligned}
$$

It is worth-noting that the multiplication of $\bar{\xi}_{i, t, t^{\prime}}$ or $\bar{\xi}_{i^{\prime}, t, t^{\prime}}$ by another binary variables also triggers the problem of non-linearity. The Glover Transformation [21] in Eqs. (11)-(14) removes this limitation by introducing new continuous auxiliary variables $\left(\Gamma_{i}, i=1, \ldots, 4\right)$ and two linear constraints for each $\Gamma i$, as follows:

$$
\begin{aligned}
& \bar{\xi}_{i, t, t^{\prime}}-V_{i}^{\max }\left(1-\bar{N}_{i, t, t^{\prime}}\right) \leqslant \Gamma_{1}\left(i, t, t^{\prime}\right) \leqslant \bar{\xi}_{i, t, t^{\prime}} \\
& 0 \leqslant \Gamma_{1}\left(i, t, t^{\prime}\right) \leqslant V_{i}^{\max } \bar{N}_{i, t, t^{\prime}} \\
& \forall i \in \mathscr{I}_{H}^{b}, t, t^{\prime} \in \mathscr{T}, t<t^{\prime} \leqslant t+\Delta t, t \neq T \\
& \bar{\xi}_{i, t, t^{\prime}}-V_{i}^{\max }\left(1-\sum_{i^{\prime} \in \mathcal{I}_{C}^{b}} x_{i, i^{\prime}, t+\delta_{i}, t^{\prime}+\delta_{i}^{\prime}}\right) \leqslant \Gamma_{2}\left(i, t, t^{\prime}\right) \leqslant \bar{\xi}_{i, t, t^{\prime}} \\
& 0 \leqslant \Gamma_{2}\left(i, t, t^{\prime}\right) \leqslant V_{i}^{\max } \sum_{i^{\prime} \in \mathscr{I}_{C}^{b}} x_{i, i^{\prime}, t+\delta_{i}, t^{\prime}+\delta_{i}^{\prime}} \\
& \forall i \in \mathscr{I}_{H}^{b}, t, t^{\prime} \in \mathscr{T}, t<t^{\prime} \leqslant t+\Delta t, t \neq T \\
& \bar{\xi}_{i^{\prime}, t, t^{\prime}}-V_{i^{\prime}}^{\max }\left(1-\bar{N}_{i^{\prime}, t, t^{\prime}}\right) \leqslant \Gamma_{3}\left(i^{\prime}, t, t^{\prime}\right) \leqslant \bar{\xi}_{i^{\prime}, t, t^{\prime}} \\
& 0 \leqslant \Gamma_{3}\left(i^{\prime}, t, t^{\prime}\right) \leqslant V_{i^{\prime}}^{\max } \bar{N}_{i^{\prime}, t, t^{\prime}} \\
& \forall i^{\prime} \in \mathscr{I}_{C}^{b}, t, t^{\prime} \in \mathscr{T}, t<t^{\prime} \leqslant t+\Delta t, t \neq T \\
& \bar{\xi}_{i^{\prime}, t, t^{\prime}}-V_{i^{\prime}}^{\max }\left(1-\sum_{i \in \mathscr{I}_{H}^{b}} x_{i, i^{\prime}, t+\delta_{i^{\prime}}, t^{\prime}+\delta_{i^{\prime}}^{\prime}}\right) \leqslant \Gamma_{4}\left(i^{\prime}, t, t^{\prime}\right) \leqslant \bar{\xi}_{i^{\prime}, t, t^{\prime}} \\
& 0 \leqslant \Gamma_{4}\left(i^{\prime}, t, t^{\prime}\right) \leqslant V_{i^{\prime}}^{\max } \sum_{i \in \mathscr{I}_{H}^{b}} x_{i, i^{\prime}, t+\delta_{i^{\prime}}, t^{\prime}+\delta_{i^{\prime}}^{\prime}} \\
& \forall i^{\prime} \in \mathscr{I}_{C}^{b}, t, t^{\prime} \in \mathscr{T}, t<t^{\prime} \leqslant t+\Delta t, t \neq T
\end{aligned}
$$

\subsubsection{Objective function}

The objective function for short-term scheduling in Eq. (21) is a slightly modified version from Castro et al. [2]. To measure the total profit, the first term calculates the income from selling products or cost from buying raw materials. The second term denotes operational cost and the third term refers to the cost for total utilities, where $Q_{H}$ and $Q_{C}$ can be calculated from Eqs. (19) and (20), representing total required steam and cooling water, respectively.

$$
\begin{aligned}
& Q_{H}=\sum_{t \in \mathscr{T}} \sum_{\substack{t^{\prime} \in \mathscr{T} \\
t<t^{\prime} \leqslant t+\Delta t}} \sum_{i \in \mathscr{I}_{H}^{b}}\left(q_{i, t, t^{\prime}}+q_{i, t, t^{\prime}}^{\prime}\right) \\
& Q_{C}=\sum_{t \in \mathscr{T}} \sum_{\substack{t^{\prime} \in \mathscr{T} \\
t<t^{\prime} \leqslant t+\Delta t}} \sum_{\substack{i^{\prime} \in \mathscr{P}_{C}^{b} \\
\left(i^{\prime}, t, t^{\prime}\right.}}\left(q_{i^{\prime}, t, t^{\prime}}\right)
\end{aligned}
$$

$$
\begin{aligned}
& \max _{\boldsymbol{y} \in \boldsymbol{\Omega}_{1}} \sum_{r \in \mathscr{R}} c_{r}\left(R_{r, T}-R_{r}^{0}\right)-\sum_{i \in \mathscr{I}} \sum_{\substack{t \in \mathscr{T} \\
t \neq T}} \sum_{\substack{t^{\prime} \in \mathscr{T} \\
t<t^{\prime} \leqslant t+\Delta t}}\left(c_{i} \bar{N}_{i, t, t^{\prime}}+\bar{c}_{i} \bar{\xi}_{i, t, t^{\prime}}\right) \\
& -\left(C_{H} Q_{H}+C_{C} Q_{C}\right)
\end{aligned}
$$

$\boldsymbol{y}$ is the variable vector $\left(\bar{N}_{i, t, t^{\prime}}, x_{i, i^{\prime}, t, t^{\prime}}, R_{r, t}, T_{t}, \bar{\xi}_{i, t, t^{\prime}}\right)$ and $\boldsymbol{\Omega}_{1}$ denotes the feasible searching space defined by all constraints, Eqs. (1)-(20).

\subsection{Formulation for periodic scheduling problems}

Most of the constraints for periodic scheduling problems are inherited from those for short-term scheduling. In periodic sched- uling formulation, the end time point $T$ should be mapping to the time point 1 because the termination of a cycle must become the beginning point of the subsequent cycle. Conveniently to express a relative time point $t^{\prime}$ to the corresponding time point $t$, a wrapping operator $\Phi(t)$ is introduced, defined as a pointer to the actual time point as shown in Eq. (22).

$$
\Phi(t)= \begin{cases}t-T+1 & \text { for } t \geqslant T \\ t & \text { for } 1 \leqslant t \leqslant T \\ t+T-1 & \text { for } t<1\end{cases}
$$

\subsubsection{Time constraints}

If a task might be terminated at the next cycle, the original timing constraint must be decomposed into two parts. The first part denotes the situation when $T_{t^{\prime}}$ is larger than $T_{t}$ (Eqs. (23) and (24)) whereas the second part is preserved for the scenario that the time point $t^{\prime}$ rolls back to a position earlier than $t$ (Eqs. (25) and (26)). As those in short-term formulation, Eqs. (23) and (25) consist all tasks while Eqs. (24) and (26) only involve the zero-wait tasks. It must be noted that we always use the wrapping operator in the expression of heat-integration binary variable combination. This is because it is uncertain whether the time point $t+\delta_{i}$ or $t^{\prime}+\delta_{i}^{\prime}$ will extend to next cycle or not. Thus the wrapping operator serves as a general time point converter in time point shifting.

$$
\begin{aligned}
T_{t^{\prime}}-T_{t} \geqslant \sum_{i \in \mathscr{I}^{b}} \bar{\mu}_{r, i}\left[\alpha_{i} \bar{N}_{i, t, t^{\prime}}+\Delta \alpha_{i}\left(\sum_{i^{\prime} \in \mathscr{S}_{C}^{b}} x_{i, i^{\prime}, \Phi\left(t+\delta_{i}\right), \Phi\left(t^{\prime}+\delta_{i}^{\prime}\right)}\right)\right. \\
\left.\left.+\sum_{i^{\prime} \in \mathscr{S}_{H}^{b}} x_{i^{\prime}, i, \Phi\left(t+\delta_{i}\right), \Phi\left(t^{\prime}+\delta_{i}^{\prime}\right)}\right)+\beta_{i} \bar{\xi}_{i, t, t^{\prime}}\right] \\
\forall r \in \mathscr{R}^{\mathrm{EQ}}, t, t^{\prime} \in \mathscr{T}, t<t^{\prime} \leqslant t+\Delta t, t^{\prime}<T \\
\left(T_{t^{\prime}}+H\right)-T_{t} \geqslant \sum_{i \in \mathscr{I}^{b}} \bar{\mu}_{r, i}\left[\alpha_{i} \bar{N}_{i, t, t^{\prime}}+\Delta \alpha_{i}\left(\sum_{i^{\prime} \in \mathscr{I}_{C}^{b}} x_{i, i^{\prime}, \Phi\left(t+\delta_{i}\right), \Phi\left(t^{\prime}+\delta_{i}^{\prime}\right)}\right.\right. \\
\left.\left.+\sum_{i^{\prime} \in \mathscr{I}_{H}^{b}} x_{i^{\prime}, i, \Phi\left(t+\delta_{i}\right), \Phi\left(t^{\prime}+\delta_{i}^{\prime}\right)}\right)+\beta_{i} \bar{\xi}_{i, t, t^{\prime}}\right] \\
\forall r \in \mathscr{R}^{\mathrm{EQ}}, t, t^{\prime} \in \mathscr{T}, t^{\prime} \leqslant t+\Delta t-T+1, t<T
\end{aligned}
$$

$$
\begin{aligned}
T_{t^{\prime}}-T_{t} \leqslant & H^{\max }\left(1-\sum_{i \in \mathscr{J}^{Z W}} \bar{\mu}_{r, i} \bar{N}_{i, t, t^{\prime}}\right) \\
& +\sum_{i \in \mathscr{I}^{Z W}} \bar{\mu}_{r, i}\left[\alpha_{i} \bar{N}_{i, t, t^{\prime}}+\Delta \alpha_{i}\left(\sum_{i^{\prime} \in \mathscr{S}_{\mathcal{C}}^{b} \wedge I^{Z W}} x_{i, i^{\prime}, \Phi\left(t+\delta_{i}\right), \Phi\left(t^{\prime}+\delta_{i}^{\prime}\right)}\right.\right. \\
& \left.\left.+\sum_{i^{\prime} \in \mathscr{I}_{H}^{b} \wedge I^{Z W}} x_{i^{\prime}, i, t, t^{\prime}}\right)+\beta_{i} \bar{\xi}_{i, t, t^{\prime}}\right] \\
& \forall r \in \mathscr{R}^{\mathrm{EQ}}, t, t^{\prime} \in \mathscr{T}, t<t^{\prime} \leqslant t+\Delta t, t^{\prime}<T
\end{aligned}
$$

$$
\begin{aligned}
\left(T_{t^{\prime}}+H\right)-T_{t} \leqslant & H^{\max }\left(1-\sum_{i \in \mathscr{I}^{Z W}} \bar{\mu}_{r, i} \bar{N}_{i, t, t^{\prime}}\right) \\
& +\sum_{i \in \mathscr{I}^{Z W}} \bar{\mu}_{r, i}\left[\alpha_{i} \bar{N}_{i, t, t^{\prime}}+\Delta \alpha_{i}\left(\sum_{i^{\prime} \in \mathscr{I}_{C}^{b} \wedge I^{Z W}} x_{i, i^{\prime}, \Phi\left(t+\delta_{i}\right), \Phi\left(t^{\prime}+\delta_{i}^{\prime}\right)}\right)+\beta_{i} \bar{\xi}_{i, t, t^{\prime}}\right] \\
& \left.+\sum_{i^{\prime} \in \mathscr{I}_{H}^{b} \wedge I^{Z W}} x_{i^{\prime}, i, t, t^{\prime}}\right) \\
& \forall r \in \mathscr{R} \\
& \mathrm{EQ}, t, t^{\prime} \in \mathscr{T}, t^{\prime} \leqslant t+\Delta t-T+1, t<T
\end{aligned}
$$


The absolute time of the first time point is still fixed as zero.

$T_{1}=0$

Eq. (28) is integrated into the formulation to speed up the problem solving.

$T_{t} \leqslant H^{\max } \quad \forall t \in \mathscr{T}, t \neq T$

In the periodic scheduling case, we do not really access any information at time point $T$. However, the definition of the last time point as cycle time will make a comparable formulation with the short-term case which means time horizon.

$T_{T}=H$

Unlike the corresponding confinement in Eq. (5), (30) plays a more important role in periodic scheduling problem because the cycle time is usually a variable.

$H^{\min } \leqslant H \leqslant H^{\max }$

\subsubsection{Operational constraints}

Because the end of a task could expand beyond cycle, it is necessary to include these constraints in Eq. (31).

$V_{i}^{\min } \bar{N}_{i, t, t^{\prime}} \leqslant \bar{\xi}_{i, t, t^{\prime}} \leqslant V_{i}^{\max } \bar{N}_{i, t, t^{\prime}} \quad \forall i \in \mathscr{I}^{b}, t, t^{\prime} \in \mathscr{T}$,

$t<t^{\prime} \leqslant t+\Delta t, t^{\prime}<T \vee t^{\prime} \leqslant t+\Delta t-T+1, t<T$

\subsubsection{Excess resource balance constraints}

The excess resource balance defined in Eq. (32) is a match for Eq. (7). However, since the key issue of periodic formulation is to obtain an optimal repetition, the general resource balance is valid only for the equipment resources unless we also consider material's demand rate for material related resources (raw material, final product and intermediate). Therefore, in Eq. (32), the demand rate is once time in each periodic cycle.

$$
\begin{aligned}
& R_{r, t}=R_{r, \Phi(t-1)}+\sum_{i \in \mathscr{I}^{b}}\left[\sum_{\substack{t^{\prime} \in \mathscr{T} \\
t<t^{\prime} \leqslant t+\Delta t, t^{\prime}<T \\
t^{\prime} \leqslant t+\Delta t-T+1, t<T}}\left(\mu_{r, i} \bar{N}_{i, t, t^{\prime}}+v_{r, i} \bar{\xi}_{i, t, t^{\prime}}\right)\right. \\
& \left.+\sum_{\substack{t^{\prime} \in \mathcal{F} \\
t-\Delta t \leq t^{\prime}<t, t<T \\
t^{\prime} \geqslant t-\Delta t+T-1, t^{\prime}<T}}\left(\bar{\mu}_{r, i} \bar{N}_{i, t^{\prime}, t}+\bar{v}_{r, i} \bar{\xi}_{i, t^{\prime}, t}\right)\right] \\
& +\left.\Delta_{r}\right|_{t=1, r \in \mathscr{R}^{\mathrm{RM}}}-\left.\Delta_{r}\right|_{t=1, r \in \mathscr{R}^{\mathrm{FP}}} \quad \forall r \in \mathscr{R}, t \in \mathscr{T}
\end{aligned}
$$

3.2.3.1. Total mass balance. Eq. (33) represents the total equipment balance at the final time point $T-1$ (it is important to bear in mind that the time point $T$ is not the final time point but refers to time point 1). The first term of summation involves all tasks that begin from any time point; whereas the second term of summation involves those tasks that end before $T$, i.e., the same cycle.

$$
\begin{aligned}
R_{r, T-1}= & \left.R_{r}^{0}\right|_{t=1}+\sum_{i \in \mathscr{T}^{b}} \sum_{t \in T}\left(\sum_{\substack{t^{\prime} \in \mathscr{T}, t<t^{\prime} \leqslant t+\Delta t, t^{\prime}<T \\
t^{\prime} \leqslant t+\Delta t-T+1, t<T}} \mu_{r, i} \bar{N}_{i, t, t^{\prime}}\right. \\
& \left.+\sum_{\substack{t^{\prime} \in \mathscr{T} \\
t<t^{\prime} \leqslant t+\Delta t, t^{\prime}<T}} \bar{\mu}_{r, i} \bar{N}_{i, t, t^{\prime}}\right) \forall r \in \mathscr{R}^{\mathrm{EQ}}
\end{aligned}
$$

\subsubsection{Capacity constraints}

As just those in short-term case, the excess properties must lie within the given bounds.

$$
R_{r}^{\min } \leqslant R_{r, t} \leqslant R_{r}^{\max } \quad \forall r \in \mathscr{R}, t \in \mathscr{T}
$$

\subsubsection{Demand constraints}

In general, only the production rate is what we pay attention to. If a constant demand is preferred, it will give the upper and lower bounds with the same value. Eq. (35) can also set the upper bound for raw materials or intermediates if there exists certain limitation in raw supplying or waste discharging.

$\Delta_{r}^{\min } \leqslant \Delta_{r} \leqslant \Delta_{r}^{\max } \quad \forall r \in \mathscr{R}^{\mathrm{RM}}$

\subsubsection{Heat-integration constraints}

The heat-integration constraints in periodic operation are basically derived from the short-term case. The formulation difference between the two kinds of operations is that the termination of a task could be located at the next cycle. Another difference is that we use the wrapping operator in representing the starting and end time points in the binary variable $x$.

3.2.6.1. One-to-one constraints. Eqs. (36) and (37) are the periodic version of the one-to-one constraints.

$$
\begin{array}{r}
\sum_{i^{\prime} \in \mathscr{I}_{C}^{b}} x_{i, i^{\prime}, \Phi\left(t+\delta_{i}\right), \Phi\left(t^{\prime}+\delta_{i}^{\prime}\right)} \leqslant \bar{N}_{i, t, t^{\prime}} \quad \forall i \in \mathscr{I}_{H}^{b}, t, t^{\prime} \in \mathscr{T}, \\
t<t^{\prime} \leqslant t+\Delta t \vee t^{\prime} \leqslant t+\Delta t-T+1, t<T \\
\sum_{i \in \mathscr{I}_{H}^{b}} x_{i, i^{\prime}, \Phi\left(t+\delta_{i}\right), \Phi\left(t^{\prime}+\delta_{i}^{\prime}\right)} \leqslant \bar{N}_{i^{\prime}, t, t^{\prime}} \quad \forall i^{\prime} \in \mathscr{I}_{C}^{b}, t, t^{\prime} \in \mathscr{T}, \\
t<t^{\prime} \leqslant t+\Delta t \vee t^{\prime} \leqslant t+\Delta t-T+1, t<T
\end{array}
$$

3.2.6.2. External utility requirement. The computation of the external utilities required for heating or cooling tasks are shown in Eqs. (38)-(41), respectively. To linearize constraints, Glover transformation are also applied to the periodic case in the same manner. Thus, the detailed manipulation of variables is not repeated here.

Heat duty required in task $i$ at time point $t$ to $t^{\prime}$ (standalone mode)

$$
\begin{aligned}
q_{i, t, t^{\prime}}= & K_{1, i} \alpha_{i}\left(\bar{N}_{i, t, t^{\prime}}-\sum_{i^{\prime} \in \mathscr{S}_{C}^{b}} x_{i, i^{\prime}, \Phi\left(t+\delta_{i}\right), \Phi\left(t^{\prime}+\delta_{i}^{\prime}\right)}\right) \\
+ & K_{2, i} \alpha_{i} \bar{\xi}_{i, t, t^{\prime}}\left(\bar{N}_{i, t, t^{\prime}}-\sum_{i^{\prime} \in \mathscr{I}_{C}^{b}} x_{i, i^{\prime}, \Phi\left(t+\delta_{i}\right), \Phi\left(t^{\prime}+\delta_{i}^{\prime}\right)}\right) \\
& \forall i \in \mathscr{I}_{H}^{b}, t, t^{\prime} \in \mathscr{T}, t<t^{\prime} \leqslant t+\Delta t, \\
& t^{\prime}<T \vee t^{\prime} \leqslant t+\Delta t-T+1, t<T
\end{aligned}
$$

Heat duty required in task $i$ at time point $t$ to $t^{\prime}$ (integrated mode)

$$
\begin{aligned}
q_{i, t, t^{\prime}}^{\prime}= & K_{1, i}^{\prime}\left(\alpha_{i}+\Delta \alpha^{\prime}\right) \sum_{i^{\prime} \in \mathscr{I}_{C}^{b}} x_{i, i^{\prime}, \Phi\left(t+\delta_{i}\right), \Phi\left(t^{\prime}+\delta_{i}^{\prime}\right)} \\
+ & K_{2, i}^{\prime}\left(\alpha_{i}+\Delta \alpha^{\prime}\right) \bar{\xi}_{i, t, t^{\prime}} \sum_{i^{\prime} \in \mathscr{I}_{C}^{b}} x_{i, i^{\prime}, \Phi\left(t+\delta_{i}\right), \Phi\left(t^{\prime}+\delta_{i}^{\prime}\right)} \\
& \forall i \in \mathscr{I}_{H}^{b}, t, t^{\prime} \in \mathscr{T}, t<t^{\prime} \leqslant t+\Delta t, \\
& t^{\prime}<T \vee t^{\prime} \leqslant t+\Delta t-T+1, t<T
\end{aligned}
$$

Cold duty required in task $i^{\prime}$ at time point $t$ to $t^{\prime}$ (standalone mode) 


$$
\begin{aligned}
q_{i^{\prime}, t, t^{\prime}}= & K_{1, i^{\prime}} \alpha_{i^{\prime}}\left(\bar{N}_{i^{\prime}, t, t^{\prime}}-\sum_{i \in \mathscr{I}_{H}^{b}} x_{i, i^{\prime}, \Phi\left(t+\delta_{i^{\prime}}\right), \Phi\left(t^{\prime}+\delta_{i^{\prime}}^{\prime}\right)}\right) \\
+ & K_{2, i^{\prime}} \alpha_{i^{\prime}} \bar{\xi}_{i^{\prime}, t, t^{\prime}}\left(\bar{N}_{i^{\prime}, t, t^{\prime}}-\sum_{i \in \mathscr{I}_{H}^{b}} x_{i, i^{\prime},, \Phi\left(t+\delta_{i^{\prime}}\right), \Phi\left(t^{\prime}+\delta_{i^{\prime}}^{\prime}\right)}\right) \\
& \forall i^{\prime} \in \mathscr{I}_{C}^{b}, t, t^{\prime} \in \mathscr{T}, t<t^{\prime} \leqslant t+\Delta t, \\
& t^{\prime}<T \vee t^{\prime} \leqslant t+\Delta t-T+1, t<T
\end{aligned}
$$

Cold duty required in task $i^{\prime}$ at time point $t$ to $t^{\prime}$ (integrated mode)

$$
\begin{aligned}
& q_{i^{\prime}, t, t^{\prime}}^{\prime}= K_{1, i^{\prime}}^{\prime}\left(\alpha_{i^{\prime}}+\Delta \alpha^{\prime}\right) \sum_{i \in \mathscr{I}_{H}^{b}} x_{i, i^{\prime}, \Phi\left(t+\delta_{i^{\prime}}\right), \Phi\left(t^{\prime}+\delta_{i^{\prime}}^{\prime}\right)} \\
&+ K_{2, i^{\prime}}^{\prime}\left(\alpha_{i^{\prime}}+\Delta \alpha^{\prime}\right) \bar{\xi}_{i^{\prime}, t, t^{\prime}} \sum_{i \in \mathscr{I}_{H}^{b}} x_{i, i^{\prime}, \Phi\left(t+\delta_{i^{\prime}}\right), \Phi\left(t^{\prime}+\delta_{i^{\prime}}^{\prime}\right)} \\
& \forall i^{\prime} \in \mathscr{I}_{C}^{b}, t, t^{\prime} \in \mathscr{T}, t<t^{\prime} \leqslant t+\Delta t \\
& t^{\prime}<T \vee t^{\prime} \leqslant t+\Delta t-T+1, t<T
\end{aligned}
$$

\subsubsection{Objective function}

The objective function for periodic scheduling, which stands on a long-term basis is a little different from that of the short-term case. In the long run, the objective function should be defined as Eq. (44), which is the maximization of the profit for each cycle. In the objective, the total required steam and cooling water are shown in Eqs. (42) and (43), respectively.

$$
\begin{aligned}
& Q_{H}=\sum_{t \in \mathscr{T}} \sum_{\substack{t^{\prime} \in \mathscr{T}, t<t^{\prime} \leqslant t+\Delta t, t^{\prime}<T \\
t^{\prime} \leqslant t+\Delta t-T+1, t<T}} \sum_{i \in \mathscr{I}_{H}^{b}}\left(q_{i, t, t^{\prime}}+q_{i, t, t^{\prime}}^{\prime}\right) \\
& Q_{C}=\sum_{t \in \mathscr{T}} \sum_{\substack{t^{\prime} \in \mathscr{T}, t<t^{\prime} \leqslant t+\Delta t, t^{\prime}<T \\
t^{\prime} \leqslant t+\Delta t-T+1, t<T}} \sum_{\substack{i^{\prime} \in \mathscr{S}_{C}^{b} \\
t_{C}}}\left(q_{i^{\prime}, t, t^{\prime}}+q_{i^{\prime}, t, t^{\prime}}^{\prime}\right)
\end{aligned}
$$

$$
\begin{aligned}
& \max _{\boldsymbol{y} \in \boldsymbol{\Omega}_{2}}\left[\sum _ { r \in \mathscr { R } } c _ { r } \left(\left.\Delta_{r}\right|_{r \in \mathscr{R}} \sum_{i \in \mathscr{T}} \sum_{t \in \mathscr{T}} \sum_{\substack{\mathrm{FP}^{\prime} \in \mathscr{T} \\
t<t^{\prime} \leqslant t+\Delta t, t^{\prime}<T \\
t^{\prime} \leqslant t+\Delta t-T+1, t<T}}\left(\left.c_{i}\right|_{r \in \mathscr{R}^{\mathrm{RM}}}\right)\right.\right. \\
& \left.-\bar{N}_{i, t, t^{\prime}}+\bar{c}_{i} \bar{\xi}_{i, t, t^{\prime}}\right) \sum_{r \in \mathscr{R}} \\
& \left.-\left(C_{H} Q_{H}+C_{C} Q_{C}\right)\right] / H
\end{aligned}
$$

In Eq. (44), $\boldsymbol{y}$ is the variable vector $\left(\bar{N}_{i, t, t^{\prime}}, x_{i, i^{\prime}, t, t^{\prime}}, R_{r, t}, T_{t}, \bar{\xi}_{i, t, t^{\prime}}, \Delta r, H\right)$ and $\boldsymbol{\Omega}_{2}$ denotes all constraints for periodic operation, Eqs. (23)(43). It is worthwhile to note that this leads to a mixed-integer nonlinear program (MINLP) which is usually more difficult than the MILP because of its non-linearity as well as non-convexity. Another issue with regard to $H$ is that since the number of time point required is unknown in advance, we need a massive number of time points to guarantee the solution quality which would make the problem more difficult to solve. To cope with this problem, an alternative way is to solve the individual MILP by regarding the cycle times as parameters. This is a weakness in the periodic version of the formulation, in most cases, the heat integration is the main focus rather than finding a good cycle time. If a good cycle time is of major concern, a good way to accelerate the calculation is to exclude some unlikely cycle times because the best cycle time must be one of the linear combinations of the processing times. For example, it is reasonable to exclude those cycle times where the product completion is impossible. In ordinary cases, it is also very easy to identify whether only an integer cycle time is chosen or not. Despite of the fact that the problem is more difficult to solve, especially when the feasible upper bound of cycle time $\left(H^{\max }\right)$ is large, it is fortunate to know that applying a very large cycle time is inadequate in real cases because it is usually more risky to ensure the optimality for long time operation. It is not to deny the significance of global optimum but to emphasize the importance of setting a realizable cycle time in periodic scheduling problems.

\section{Numerical examples}

Two literature examples are used for demonstrating the wide applicability of the proposed model. Both short-term and periodic scheduling scenarios are investigated. All computations are done on a $2.0 \mathrm{GHZ}$ Pentium-M personal computer with GAMS 22.3/ CPLEX as the MILP solver [20].

\subsection{Example 1: a simple sequential batch process}

Consider the simple sequential batch process presented in Fig. 2, which was also used as illustration by Papageorgiou et al. [11] and Majozi [15] in their formulations. The process consists of three units with resource equipments, $R^{\mathrm{EQ}}$, including one reactor, one filter and one column that work in sequence. From input FeedA and FeedB, the process produces final products, Product1 and Product2, from Distiller and Waste from Filter. The processing time, maximum working capacity and the coefficients for the equipment/ material resources are depicted in the RTN diagram shown in Fig. 2 . The minimum working capacities are assigned as $15 \%$ of their maximum working capacities. To simplify, it is assumed that the costs on executing tasks $\left(c_{i}\right.$ and $\left.\bar{c}_{i}\right)$ are zero. The selling prices of products and the utility costs are listed in Table 2 . From the operation within the process, it is found that a massive energy must be adapted to Reaction and Distillation tasks. However, considering the limitation of working capacities, it is not possible to run any task only once; instead, each task should be run many times so as to make possible the energy exchange between Reaction and

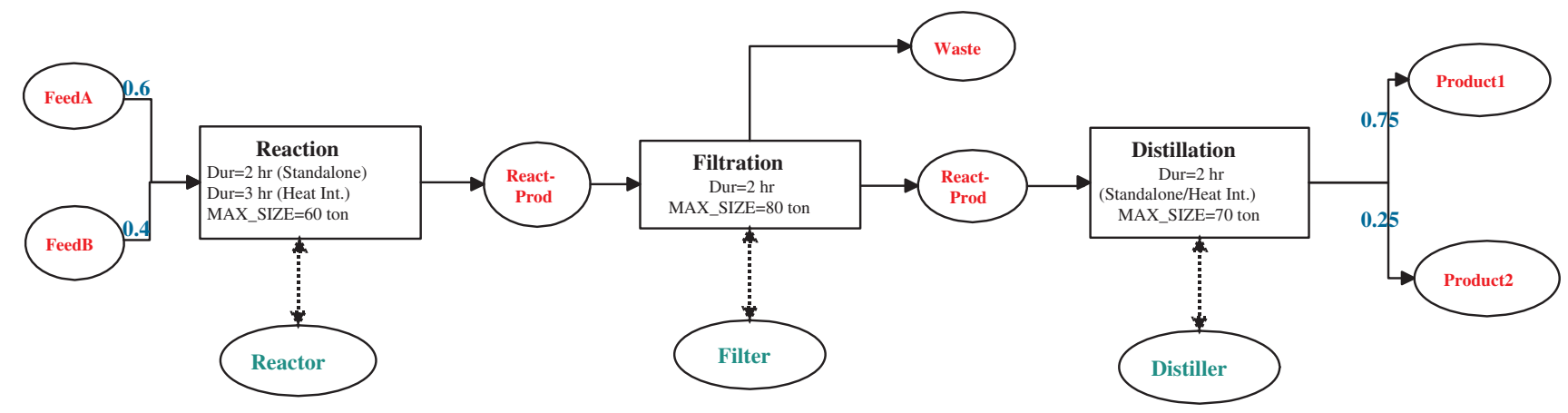

Fig. 2. RTN for example 1. 
Table 1

Basic data for example 1

\begin{tabular}{lllll}
\hline Task & Operation & Utility req. & $\begin{array}{l}\text { Amount } \\
\text { (ton/h) }\end{array}$ & $\begin{array}{l}\text { Duration } \\
(\mathrm{h})\end{array}$ \\
\hline Reaction & Standalone & Cooling water & $1.59+0.1 \bar{\xi}_{i, t, t^{\prime}}$ & 2 \\
& Heat-integrated & Cooling water & $1.0+0.060 \bar{\xi}_{i, t, t^{\prime}}$ & 3 \\
\multirow{2}{*}{ Filtration } & Standalone & None & 0 & 1 \\
Distillation & Standalone & Steam & $0.044+0.0035 \bar{\xi}_{i, t, t^{\prime}}$ & 2 \\
& Heat-integrated & Steam & $0.020+0.0016 \bar{\xi}_{i, t, t^{\prime}}$ & 2 \\
\hline
\end{tabular}

Distillation tasks. From the thermodynamics and experimental estimation, the amount of utilities is summarized in Table 1 for both standalone and heat-integrated modes. It is obvious that the required utilities is much lower in the heat-integrated mode.

The objective of this problem is to maximize the profit in $48 \mathrm{~h}$. We first test this problem by applying the proposed short-term formulation to the standalone mode optimization. Given 25 time points and $\Delta t=1$, an optimal profit of 3081.8 , the same as Papageorgiou et al. [11] and Majozi[15], is obtained for only $0.375 \mathrm{~s}$ CPU time. The scheduling result is depicted in Fig. 3 and its numerical result is shown in Table 3. Surprisingly, it is observed that the number of binary variable, 72 , is the same as that of Majozi[15]. This implies continuous time formulations in both RTN and SSN have similar benefit in reducing the problem size.

More interesting results can be found in the heat-integrated mode. Because of smaller temperature differences, the duration of Reaction in heat-integrated mode is increased from 2 to $3 \mathrm{~h}$. As mentioned in the earlier work of Papageorgiou et al. [11], the heat exchange match between task Reaction and task Distillation involves an offset of $1 \mathrm{~h}$ with respect to their starting times. To ensure that the heat-integrated tasks would always start with the

Table 2

Product selling prices and utility costs in example 1

\begin{tabular}{lllc}
\hline Product & Selling price (\$/ton) & Utility & Unit cost $(\$ /$ ton $)$ \\
\hline Product1 & 5 & Cooling water & 4 \\
Product2 & 5 & Steam & 200 \\
\hline
\end{tabular}

specified time offset, add a new constraint that enforces an alignment of $1 \mathrm{~h}$ shift from starting Reaction in order to match with Distillation as follows:

$$
\begin{aligned}
T_{t+\delta_{\text {Reaction }}} & =T_{t}+\text { offset; } \quad \text { if } x_{\text {Distillation, Reaction }, t+\delta_{\text {Reaction },}, t^{\prime}+\delta_{\text {Reaction }}^{\prime}} \\
& =1.0
\end{aligned}
$$

where the offset equals $1 \mathrm{~h}, t^{\prime}=t+1, \delta_{\text {Reaction }}=1$ and $\delta_{\text {Reaction }}^{\prime}=0$. Eq. (45) implies that it is necessary to define a time point at which the heat integration between Distillation and Reaction starts. With this connection, we can recognize from Eq. (45) that parameter $\Delta t$ must be greater than or equal to 2 because task Reaction occupies at least two time points in the heat-integrated mode.

In the first hour of Reaction in integration mode, it requires a cooling water with the amount of $1.0+0.060 \bar{\xi}_{i, t, t^{\prime}}$. However, the duration of Distillation in heat-integrated mode remains unchanged (i.e., 2 h).

In summary, 32 time points are allocated in obtaining the optimal profit of 3644.6 in 272 s CPU time. Numerical results are summarized in Table 3 . One may notice that the number of binary variable is substantially larger than the data reported by Majozi. This is because the forced alignment constraint in Eq. (45) was not involved in their work. In other word, Distillation does not consistently start 1 hour after Reaction throughout the time horizon of interest.

There is another adequate solution to handle heat-integrated problem by task decomposition. A modified RTN flowchart is expressed in Fig. 4. The feeding is virtually split into two paths: one path for standalone mode and another for heat-integrated mode. Thus two additional tasks, Reaction_STD and Reaction0, and one additional material PreR are defined. Notice that we force Reaction_STD and Reaction0 to operate in standalone mode all the time. Furthermore, Reaction0 must also zero-wait (i.e., NIS for PreR). In this case, $\Delta t$ can be 1 because the integrated/non-integrated tasks have the same processing time. As expected, the new RTN converges very fast in $0.859 \mathrm{~s}$. A summary of comparable result of both modes is given in Table 3. It is reasonable to estimate the problem size in the number of binary variable as written in Eq. (46).

$\left(N_{I}+N_{\mathrm{IBH}} N_{\mathrm{IBC}}\right) \times \frac{\Delta t\left(2 N_{T}-1-\Delta t\right)}{2}$

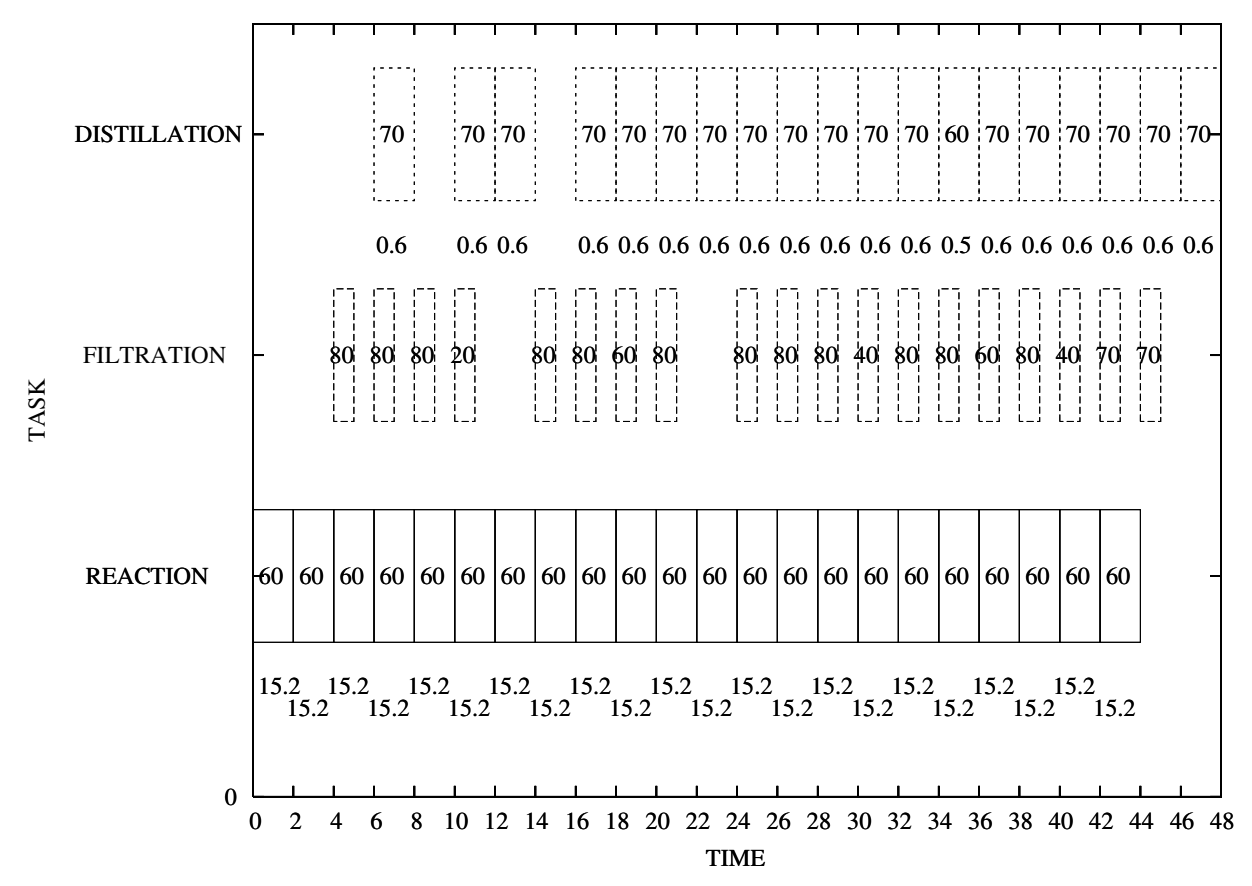

Fig. 3. Optimal schedule without heat integration for example 1 . 
Table 3

Comparative results for example 1

\begin{tabular}{|c|c|c|c|c|c|c|c|}
\hline & \multicolumn{3}{|l|}{ Standalone } & \multicolumn{4}{|l|}{ Heat-integrated } \\
\hline & Papageorgiou et al. [11] & Majozi [15] & Proposed & Papageor-giou et al. [11] & Majozi [15] & Proposed [15] & Proposed (decomp.) \\
\hline Binary variables & 142 & 72 & 72 & 188 & 96 & 244 & 186 \\
\hline Objective value & 2944.1 & 3081.8 & 3081.8 & 3644.6 & 3644.6 & 3644.6 & 3644.6 \\
\hline Product1 $(\mathrm{t})$ & 945 & 990 & 990 & 720 & 720 & 720 & 720 \\
\hline Product2 $(\mathrm{t})$ & 315 & 330 & 330 & 240 & 240 & 240 & 240 \\
\hline Steam $(\mathrm{t})$ & 10.4 & 10.9 & 10.9 & 3.6 & 3.6 & 3.6 & 3.6 \\
\hline Cooling water $(\mathrm{t})$ & 318.8 & 334 & 334 & 107.2 & 107.2 & 107.2 & 107.2 \\
\hline Margin of optimality & $5 \%$ & $0 \%$ & $0 \%$ & $5 \%$ & $<1.3 \%$ & $<5.36 \%$ & $0 \%$ \\
\hline Integrality gap & $4.76 \%$ & $0 \%$ & $0 \%$ & $0.37 \%$ & $0 \%$ & $0 \%$ & $0 \%$ \\
\hline CPU time (s) & $\mathrm{N} / \mathrm{A}$ & 24.5 & 0.375 & $\mathrm{~N} / \mathrm{A}$ & 9812.7 & 272 & 0.859 \\
\hline
\end{tabular}

where $N_{I}$ denotes the number of tasks; $N_{\text {IBH }}$ and $N_{\text {IBC }}$ mean the number of tasks that require heating and cooling, respectively; $N_{T}$ is the number of time points.

If the heat integration is considered only in the short-term scheduling case, as shown in Fig. 5, one can observe that the optimal schedule involves using the Reaction as heat source at $t=45$, i.e., the final time slot of operation, even the product of Reaction (React-Prod) is no more required. This phenomenon would easily vanish by taking the cost of operation into consideration. However, nothing will show the benefit of heat integration better than a long-term optimization profile because the cost of utilities is proportional to the overall operation time. One of the good measures is to use periodic operation (see Fig. 6).

If a periodic operation is considered, the cycle time $H$ becomes a variable that makes the model nonlinear. As mentioned earlier, one may regard the cycle time $H$ as a given parameter, keeping the problem in MILP, and optimize each sub-problem. Then select the $H$ value that has the maximum rate of profit. However, no matter which approach is applied, the upper bound of cycle time $\left(H^{\mathrm{max}}\right)$ is a very important parameter that must be given a priori. It is worthwhile to note that if a large $H^{\max }$ is applied, it is inevitable to use a large number of time points that make the MINLP more difficult to be solved. However, in this example, it is reasonable to obtain a short optimum cycle time because there is only one equipment for each task and the process is simply operated in sequence. Given a searching range from 1 to $9 \mathrm{~h}$. From the comparative results listed in Table 4 we find that the best cycle time is $3 \mathrm{~h}$ where the optimal Gantt chart is rendered in Fig. 7. This is a very reason- able result because it is more economical for the Reaction and Distillation to operate in the heat-integrated mode where $3 \mathrm{~h}$ of processing time is required. Moreover, each task uses its maximum capacity. In this connection, it can be concluded that the result depicted in Fig. 7 is also the global optimal solution, no matter how large of $H^{\max }$ is applied.

\subsection{Example 2: a process consists of five consecutive steps}

Consider a larger problem tested by Majozi [15], which is consisting of five consecutive steps as shown in Fig. 8. Materials S1 and $\mathbf{S 2}$ are conducted into $\mathbf{R 1}$ and $\mathbf{R 2}$ for the first step of reaction. The intermediate $\mathbf{S} \mathbf{2}$ from step 1 is then reacted to $\mathbf{S} \mathbf{3}$ with another material S10. The intermediate $\mathbf{S} 3$ from step 2 is then further reacted to S4, a monosodium salt solution, which is then transferred to settling step 4 for removing solid byproduct S8. The remaining monosodium salt is transferred to step 5 to remove the excess amount of water. Finally, the monosodium salt (S6) is the product. Each step consists of two identical equipments except the settling one, which consists of three settling tanks. When shown in RTN, the overall process involves 20 resources (including 11 material resources and 9 equipment resources) and 11 tasks.

The process data are listed in Tables 5 and 6. There is an opportunity for heat integration between Reaction2 (Reaction2_R3 and Reaction2_R4) and Evaporation (Evap._EV1 and Evap._EV2). The cooling load required for Reaction2 is 5 ton and the heating load required for Evaporation is 4 ton. It is assumed that the heat transfer rate remained constant when heat integration between

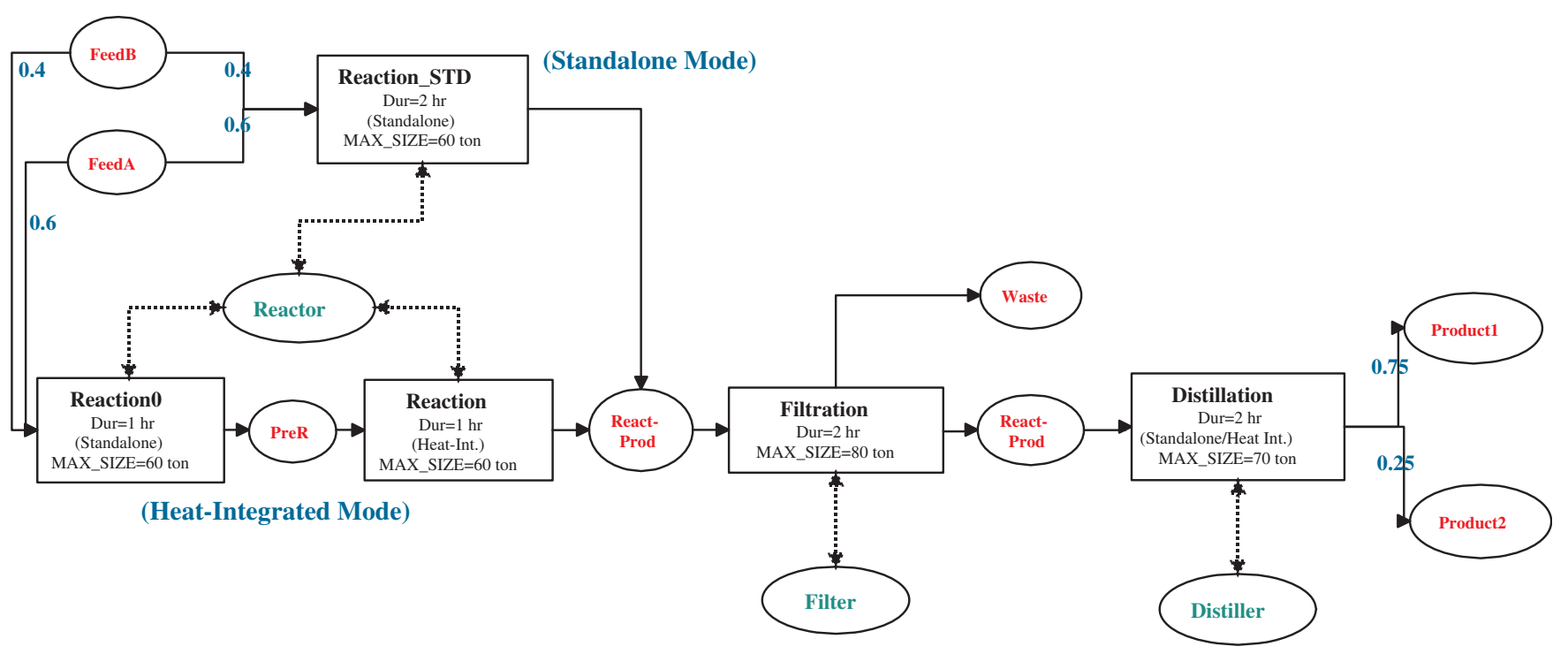

Fig. 4. Decomposed RTN for heat-integrated problem in example 1. 


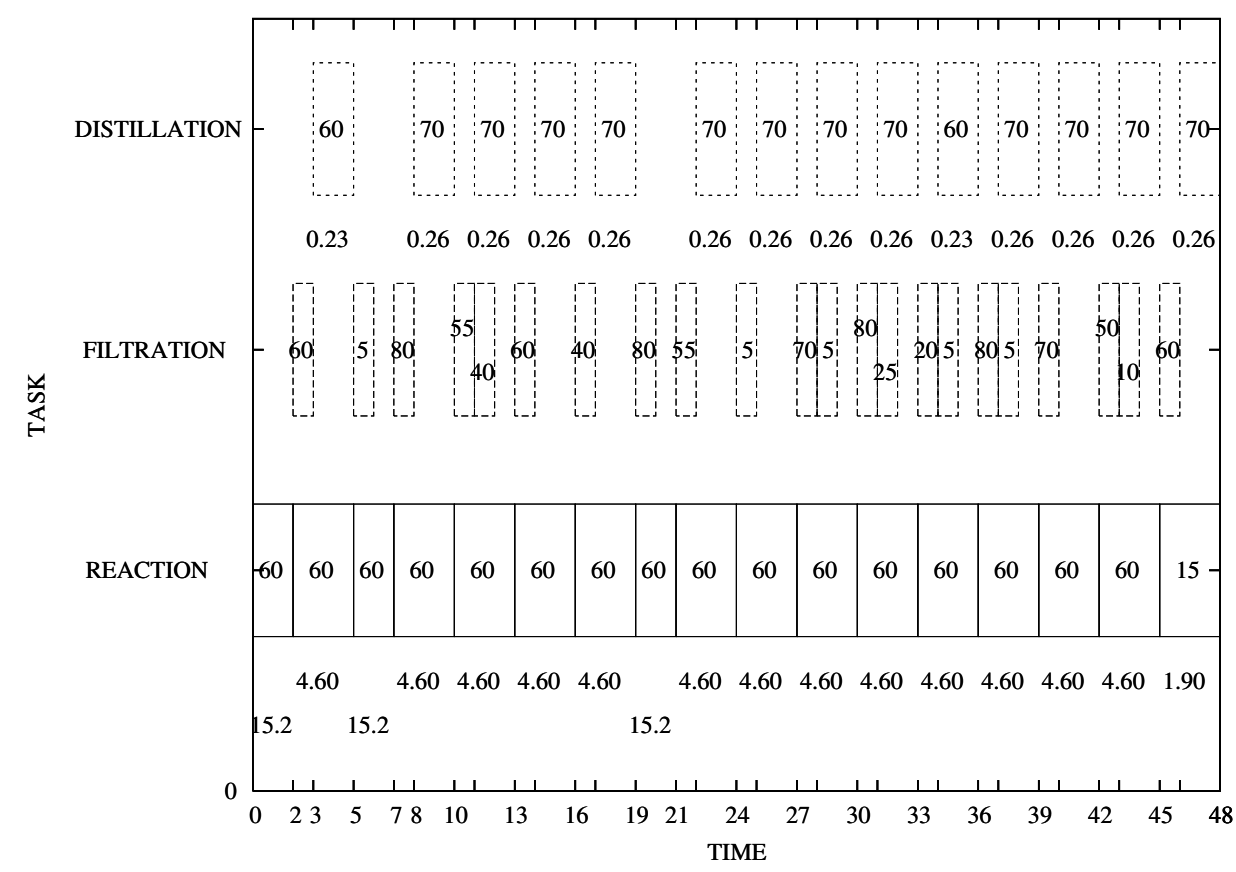

Fig. 5. Optimal schedule with heat integration for example 1.

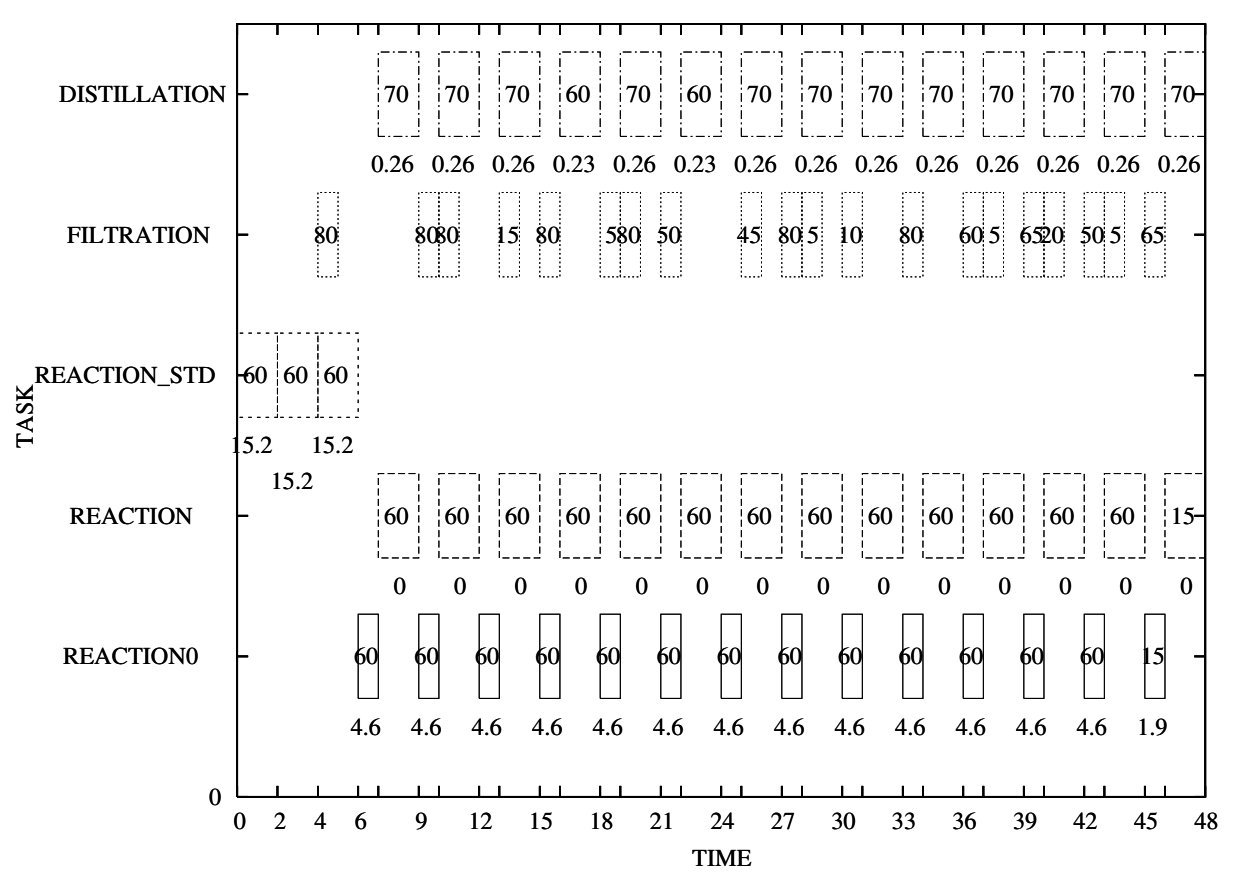

Fig. 6. Optimal schedule with heat integration for example 1 (decomposed).

Reaction2 and Evaporation takes place. Thus, in the heat-integrated mode, the external cooling load of Reaction 2 is only 1 ton while Evaporation has plenty of heat source and no steam is required. The processing time for heat and cold tasks remained unchanged $(3 \mathrm{~h})$. The selling prices of product and cost of utilities are shown in Table 7. Costs on executing tasks $\left(c_{i}\right.$ and $\left.\bar{c}_{i}\right)$ are also neglected.

First, the short-term case is investigated. Majozi[15] emphasized the scenario in the case of fixed batch size and proposed a set of simpler constraint. However, the narrowing of the formulation to a specific type problem is not of much interest in the current exploration, so we still apply the general formulation to this problem without any modification. The final Gantt diagram for
Table 4

The optimization result of fixing cycle time for example 1 in periodic scheduling mode

\begin{tabular}{lll}
\hline $\mathrm{H}$ & Profit & Profit/h \\
\hline 1 & N/A & N/A \\
2 & 137.68 & 68.84 \\
3 & 235.2 & 78.4 \\
4 & 275.36 & 68.84 \\
5 & 380.48 & 76.096 \\
6 & 470.4 & 78.4 \\
7 & 518.16 & 74.02 \\
8 & 623.28 & 77.91 \\
9 & 705 & 78.4 \\
\hline
\end{tabular}




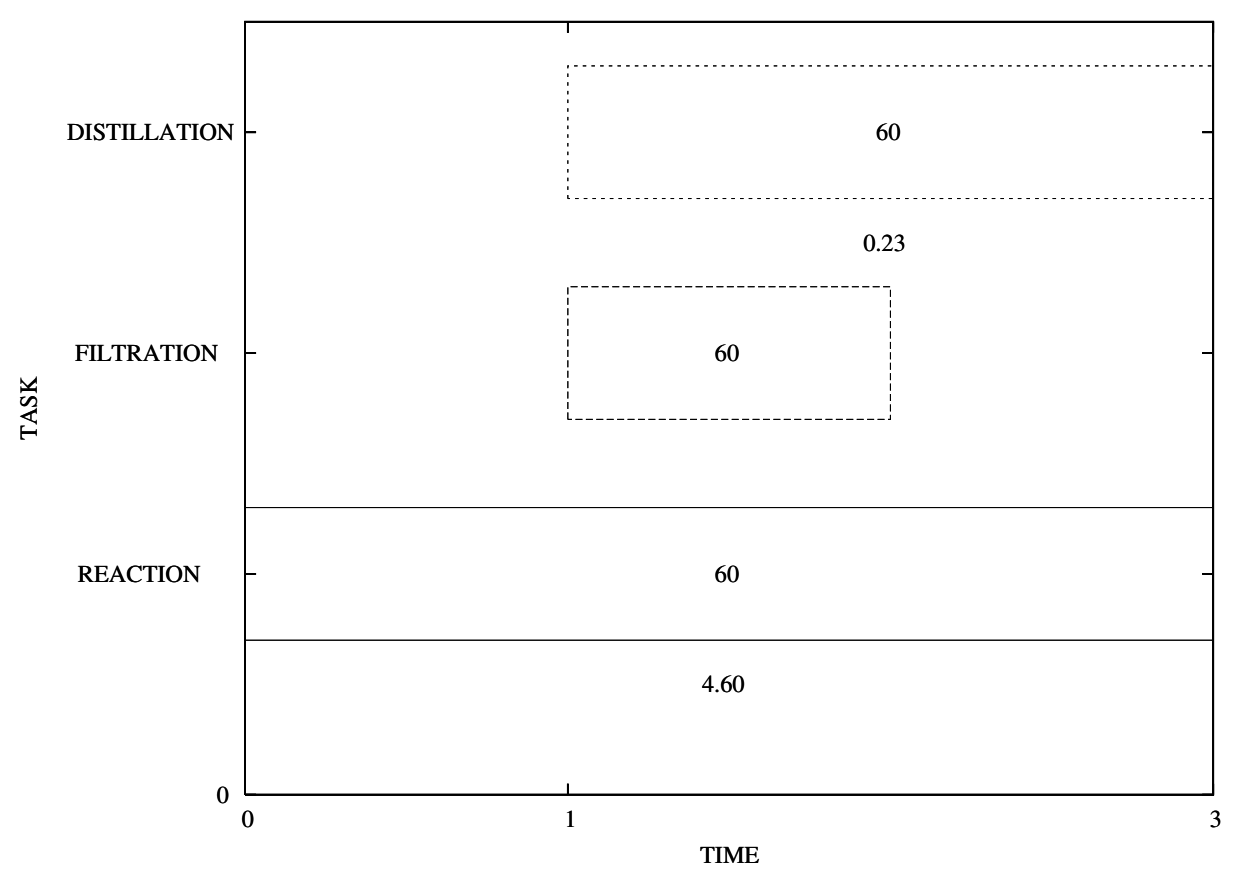

Fig. 7. Optimal periodic schedule with heat integration for example 1.

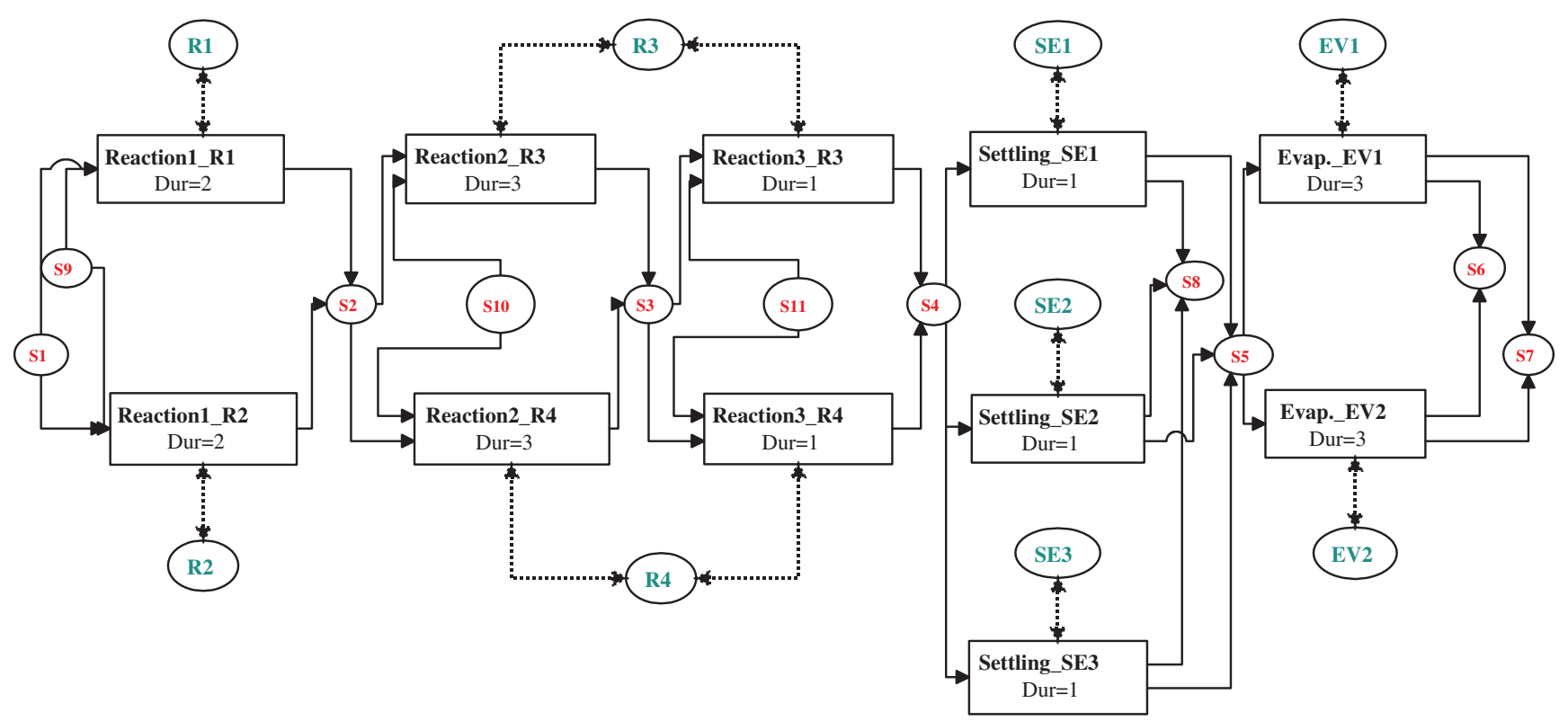

Fig. 8. RTN for example 2.

standalone and heat-integrated modes are depicted in Figs. 9 and 10. In this problem, we need $\Delta t=3$ to cope with the crossing task on 3 time points because the maximum value of processing time is threefold the minimum one. Totally 11 time points are used to find the optimal results as shown in Table 8 . One may notice that the optimal value is a little different from that of Majozi[15]. From the same value of steam and cooling water consumption, we can conclude that this inaccuracy comes from the truncation of the floating point in the stoichiometric data.

Now turn to the periodic scheduling. In comparison with the last example, the estimation of the upper bound of cycle time is more difficult because there are 2 or 3 substitutes for each kind of equipment and the problem size is bigger than that of example 1 . Given $10 \mathrm{~h}$ as the searching upper bound of cycle time, we find that it is unable to find any solution by using the literature data unless the constraint of fixed batch size is relaxed. In other words, finding a repetitive scheduling with fixed working capacities is impossible. If the minimum working capacities are assigned as $15 \%$ of their maximum working capacities (i.e., $8 \times 0.15=1.2$ ), the optimal cycle time will be $9 \mathrm{~h}$, as shown in Fig. 11. The detailed optimal cycle profit for each cycle time is compared in Table 9. Unlike the short-term scheduling result who must involve two of the Reaction2 un-integrated, all Reaction2 and Evaporation are operated in heat-integrated mode. All results in more economic use of external utilities and more profits in the long run, which provides a solid ground to apply heat integration in periodic scheduling. 
Table 5

Basic data for example 2

\begin{tabular}{|c|c|c|c|c|c|c|}
\hline Unit & Capacity & Function & Duration (h) & State & Storage capacity & Initial \\
\hline R1 & 8 & Reaction1 & 2 & s1 & Unlimited & Unlimited \\
\hline $\mathrm{R} 2$ & 8 & Reaction1 & 2 & s2 & 100 & 0 \\
\hline \multirow[t]{2}{*}{ R3 } & 8 & Reaction2 & 3 & s3 & 100 & 0 \\
\hline & 8 & Reaction3 & 1 & s4 & 100 & 0 \\
\hline \multirow[t]{2}{*}{$\mathrm{R} 4$} & 8 & Reaction2 & 3 & s5 & 100 & 0 \\
\hline & 8 & Reaction3 & 1 & s6 & 100 & 0 \\
\hline SE1 & 8 & Settling & 1 & s7 & 100 & 0 \\
\hline SE2 & 8 & Settling & 1 & s8 & 100 & 0 \\
\hline SE3 & 8 & Settling & 1 & s9 & Unlimited & Unlimited \\
\hline EV1 & 8 & Evaporation & 3 & s10 & Unlimited & Unlimited \\
\hline EV2 & 8 & Evaporation & 3 & s11 & Unlimited & Unlimited \\
\hline
\end{tabular}

Table 6

Stoichiometric data for example 2

\begin{tabular}{lll}
\hline & Ton/ton output & Ton/ton product \\
\hline s1 & 0.2 & \\
s9 & 0.25 & \\
s10 & 0.35 & \\
s11 & 0.20 & 0.7 \\
s7 & & 1 \\
s8 & & \\
\hline
\end{tabular}

Table 7

Selling prices and cost of utilities for example 2

\begin{tabular}{lllc}
\hline Product & Selling price (\$/ton) & Utility & Unit cost $(\$ /$ ton $)$ \\
\hline s6 & 100 & Cooling water & 8 \\
& & Steam & 15 \\
\hline
\end{tabular}

\section{Conclusion}

Until recently, little attention has been given to the problem of batch scheduling with direct heat integration. The aim of this work is to complement this gap. A new mixed-integer linear program (MILP) is formulated for modeling the short-term and periodic scheduling problems with direct heat integration in batch plants. The proposed formulation is based on the continuous RTN representation, which has been proved as one of the best single time grid formulation for short-term scheduling of multipurpose batch plants. In continuous RTN, since a binary variable that assigns the existence of a task contains the beginning and end points, a four-indexed binary variable for assigning heat integration is introduced naturally. In manipulating the literature examples, it is found that the new formulation gives a stricter problem description in direct heat-integration problems. This benefit is a straightforward extension from the continuous RTN model, where the heat-integration model is involved. In addition, we also attempt to generalize the heat-integration model originally proposed by Majozi [15]. For instance, a set of shifting parameter is introduced in order to handle the cases when the beginning time of heat integration is shifted from the beginning of a task. This is very useful in some arrangements, such as when pre-heating are required before the real heat integration. In this case, the processing time of a task must usually be lengthened. An adjustable parameter is also applied to handle these scenarios. These parameters will not complicate the problem but only remarkably enhance the flexibility of the formulation. Two literature examples are provided to demonstrate the novelty of the proposed formulation.

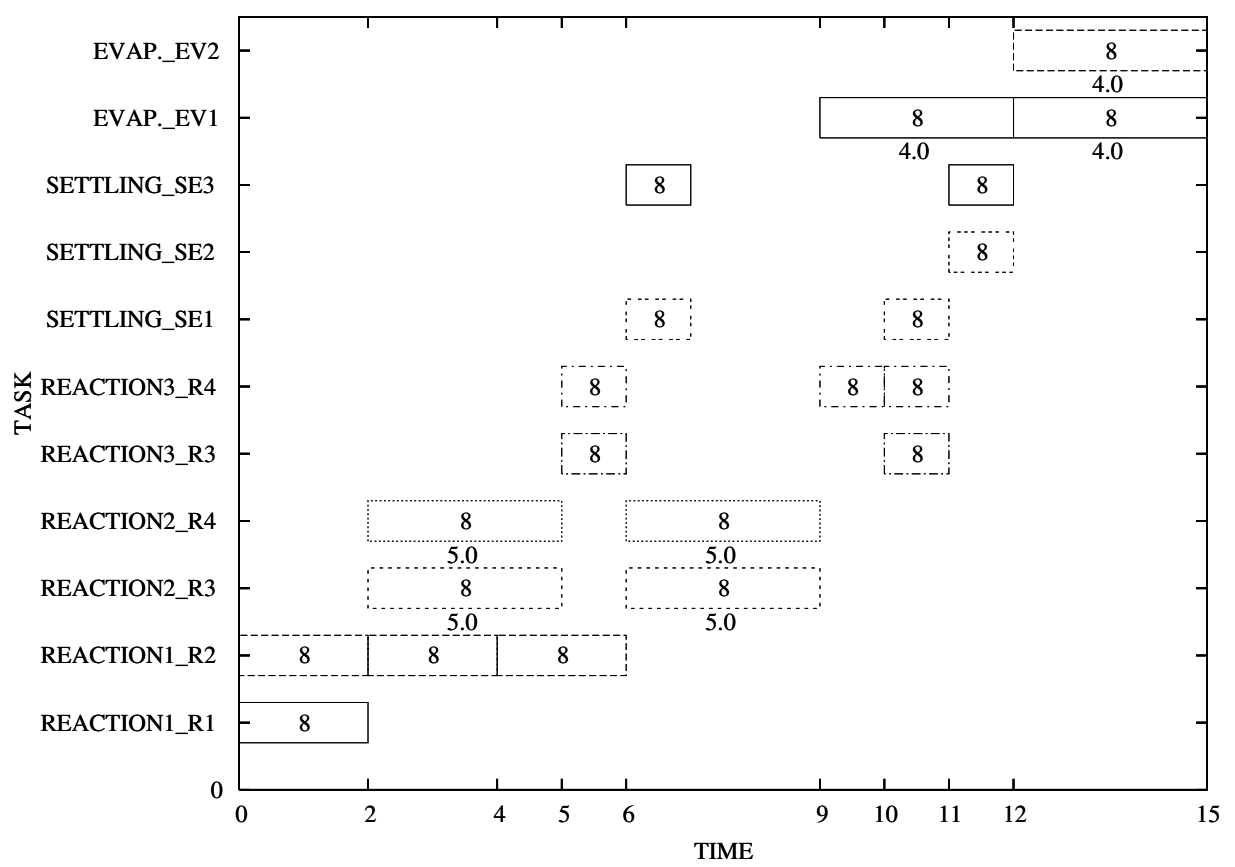

Fig. 9. Optimal schedule without heat integration for example 2 . 


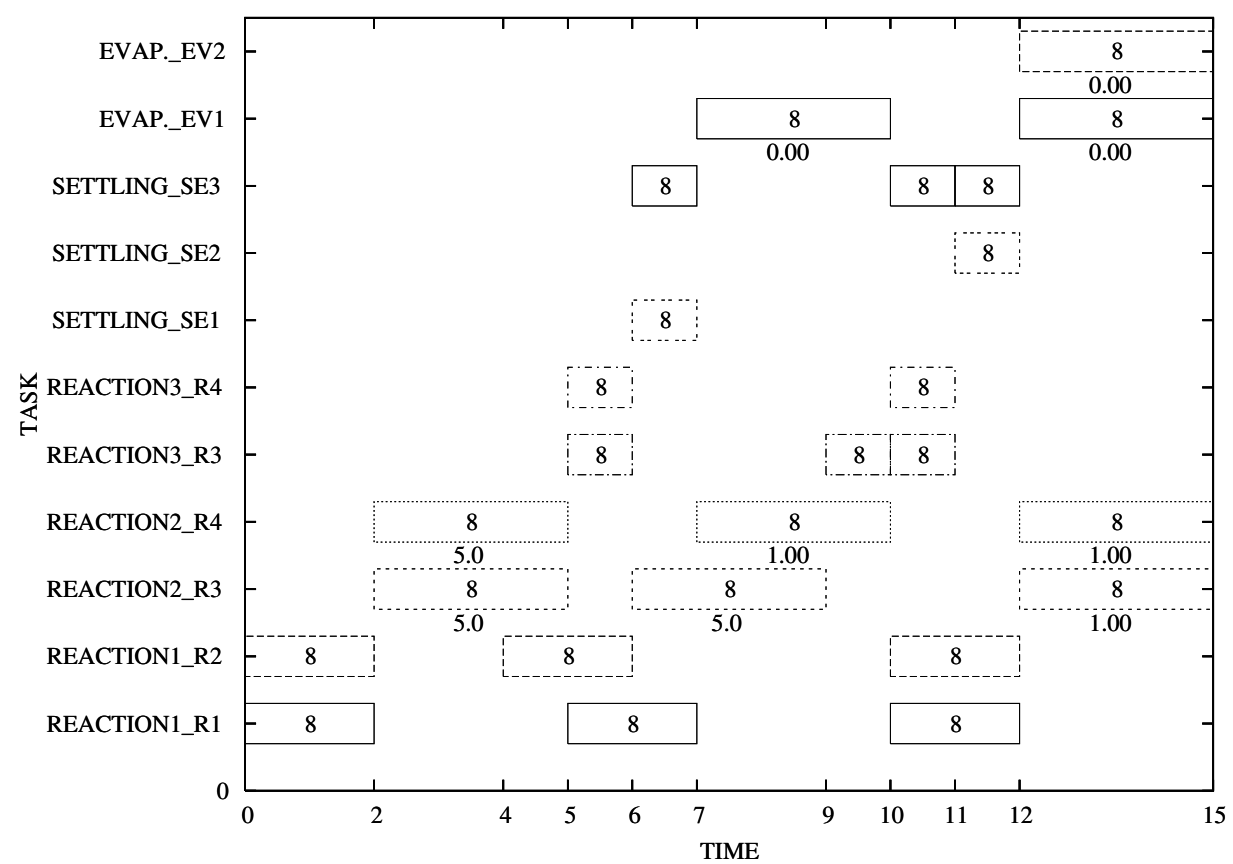

Fig. 10. Optimal schedule with heat integration for example 2.

Table 8

Comparative results for example 2

\begin{tabular}{llllll}
\hline & \multicolumn{2}{l}{ Standalone } & & \multicolumn{2}{l}{ Heat-integrated } \\
\cline { 2 - 3 } & Majozi[15] & Proposed & & Majozi[15] & Proposed \\
\hline Objective value & 1060 & 1071 & & 1256 & 1267 \\
Product $(\mathrm{t})$ & 14 & 14.1 & & 14 & 14.1 \\
Steam $(\mathrm{t})$ & 20 & 20 & & 18 & 18 \\
Cooling water $(\mathrm{t})$ & 12 & 12 & & 0 & 0 \\
Margin of optimality & $0 \%$ & $0 \%$ & & $0 \%$ & $0 \%$ \\
Integrality gap & $0 \%$ & $0 \%$ & & $0 \%$ & $0 \%$ \\
CPU time (s) & 26 & 64.67 & & 26 & 113.80 \\
\hline
\end{tabular}

Although this work mainly investigates the possibility of direct heat integration, it is also possible to include the indirect heat integration simultaneously by incorporating mass and energy balances for each time point. As mentioned by Papageorgiou et al. [11], it may lead to a non-convex MINLP. An in-depth research on extending the continuous scheduling model to the more general heatintegration problem will be of high value.

\section{Acknowledgements}

Financial supports of the Ministry of Economic Affairs (under grant 96-EC-17-A-09-S1-019) and the National Science Council of ROC (under grant NSC96-2221-E-002-151-MY3) are appreciated.

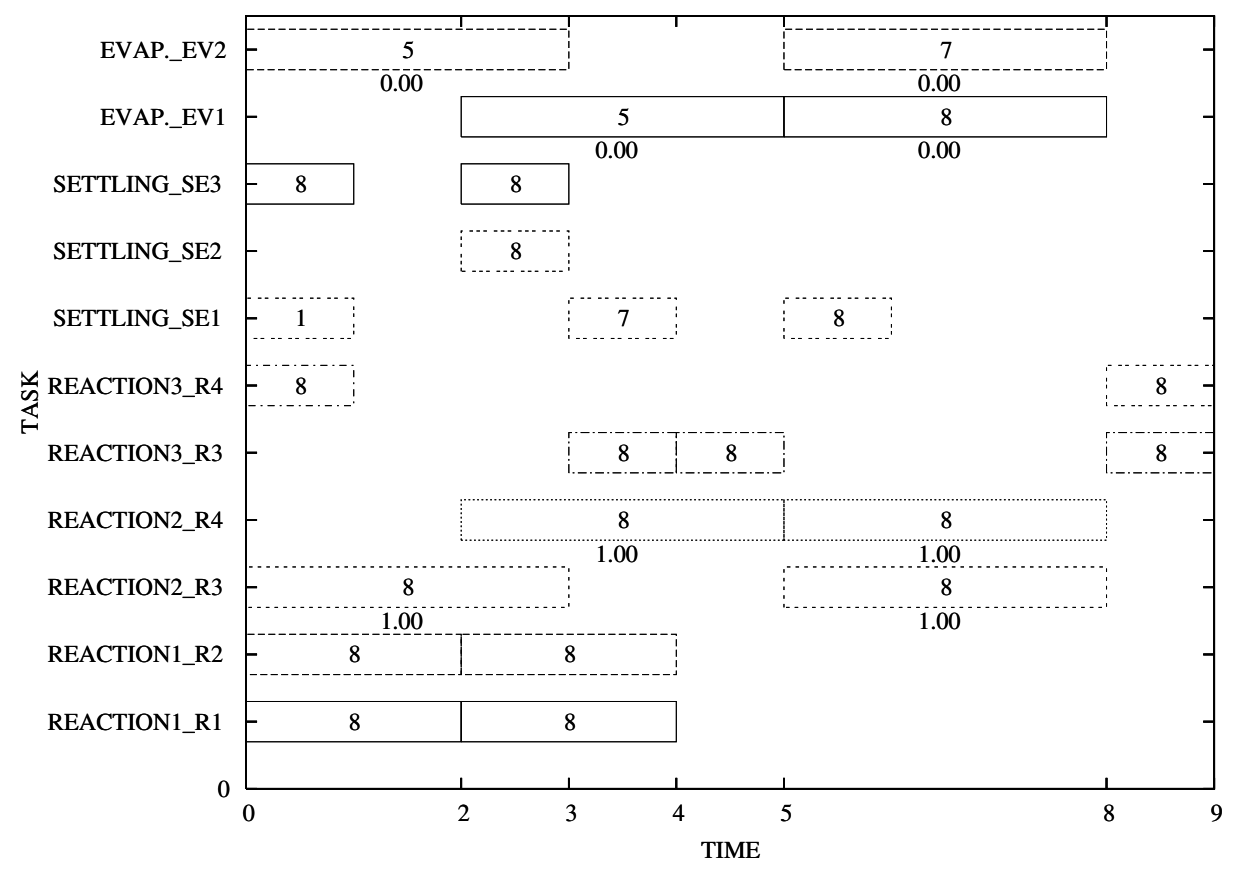

Fig. 11. Optimal periodic schedule with heat integration for example 2 . 


\section{Table 9}

The optimization results of fixing cycle time for example 2 in periodic scheduling mode

\begin{tabular}{rll}
\hline $\mathrm{H}$ & Profit & Profit $/ \mathrm{h}$ \\
\hline 2 & N/A & N/A \\
3 & 362.37 & 120.79 \\
4 & 576.59 & 144.15 \\
5 & 724.74 & 144.95 \\
6 & 864.89 & 144.15 \\
7 & 1087.11 & 155.30 \\
8 & 1153.19 & 144.15 \\
9 & 1449.48 & 161.05 \\
10 & 1449.48 & 144.95 \\
\hline
\end{tabular}

\section{References}

[1] P.M. Castro, A.P. Barbosa-Póvoa, H.A. Matos, Optimal periodic scheduling of batch plants using RTN-based discrete and continuous-time formulations: a case study approach, Ind. Eng. Chem. Res. 42 (2003) 3346-3360.

[2] P.M. Castro, A.P. Barbosa-Póvoa, H.A. Matos, A.Q. Novais, Simple continuoustime formulation for short-term scheduling of batch and continuous processes, Ind. Eng. Chem. Res. 43 (2004) 105-118.

[3] B. Lee, R.V. Reklaitis, Optimal scheduling of cyclic batch processes for heat integration-I. basic formulation, Comput. Chem. Eng. 19 (8) (1995) 883-905.

[4] N. Vaklieva-Bancheva, B.B. Ivanov, A new approach for determination of the horizon constraints for design problem of multipurpose batch chemical plants, Comput. Chem. Eng. S17 (1993) S21-S26.

[5] I.C. Kemp, Pinch Analysis and Process Integration, Elsevier, Amsterdam, 2007.

[6] J.A. Vaselenak, I.E. Grossmann, A.W. Westerberg, Heat integration in batch processing, Ind. Eng. Chem. Process Des. Dev. 25 (1986) 357-366.

[7] N. Vaklieva-Bancheva, B.B. Ivanov, N. Shah, C.C. Pantelides, Heat exchanger network design for multipurpose batch plants, Comput. Chem. Eng. 20 (8) (1996) 989-1001.
[8] M. Bozan, F. Borak, I. Or, A computerized and integrated approach for heat exchanger network design in multipurpose batch plants, Chem. Eng. Process. 40 (2001) 511V524.

[9] I.C. Kemp, A.W. Deakin, The cascade analysis for energy and process integration of batch processes, Part 1: calculation of energy targets, Chem. Eng. Res. Des. 67 (1989) 495-509.

[10] S.H. Jung, I.B. Lee, D.R. Yang, K.S. Chang, Synthesis of maximum energy recovery networks in batch processes, Korean J. Chem. Eng. 11 (3) (1994) 162171.

[11] L.G. Papageorgiou, N. Shah, C.C. Pantelides, Optimal scheduling of heatintegrated multipurpose plants, Ind. Eng. Chem. Res. 33 (1994) 3168-3186.

[12] E. Kondili, C.C. Pantelides, R.W.H. Sargent, A general algorithm for short-term scheduling of batch operations. Part I - mathematical formulation, Comput. Chem. Eng. 17 (1993) 211-227.

[13] A.P. Barbosa-Póvoa, T. Pinto, A.Q. Novais, Optimal design of heat-integrated multipurpose batch facilities: a mixed-integer mathematical formulation, Comput. Chem. Eng. 25 (2001) 547-559.

[14] T. Pinto, A.P. Barbosa-Póvoa, A.Q. Novais, Optimal design of heat-integrated multipurpose batch facilities with economic savings in utilities: a mixed integer mathematical formulation, Ann. Oper. Res. 120 (2003) 201-230.

[15] T. Majozi, Heat integration of multipurpose batch plants using a continuoustime framework, Appl. Therm. Eng. 26 (2006) 1369-1377.

[16] T. Majozi, X.X. Zhu, A novel continuous-time MILP formulation for multipurpose batch plants. 1. Short-term scheduling, Ind. Eng. Chem. Res. 40 (2001) 5935-5949.

[17] M.A. Shaik, S.L. Janak, C.A. Floudas, Continuous-time models for short-term scheduling of multipurpose Batch plants: a comparative study, Ind. Eng. Chem. Res. 45 (2006) 6190-6209.

[18] C.A. Floudas, X. Lin, Continuous-time versus discrete-time approaches for scheduling of chemical processes: a review, Comput. Chem. Eng. 28 (2004) 2109-2129.

[19] C.A. Méndez, J. Cerda, I.E. Grossmann, I. Harjunkoski, M. Fahl, State-of-the-art review of optimization methods for short-term scheduling of batch processes, Comput. Chem. Eng. 30 (2006) 913-946.

[20] A. Brooke, D. Kendrick, A. Meeraus, R. Raman, R.E. Rosenthal, GAMS: A User's Guide, Scientific Press, Redwood city, 2003.

[21] F. Glover, Improved linear integer programming formulations of nonlinear integer problems, Manage. Sci. 22 (4) (1975) 455-460. 